\title{
MULTI-WAVELENGTH OBSERVATIONS OF SUPERNOVA 2011ei: TIME-DEPENDENT CLASSIFICATION OF TYPE IIb AND Ib SUPERNOVAE AND IMPLICATIONS FOR THEIR PROGENITORS
}

\author{
Dan Milisavljevic ${ }^{1}$, Raffaella Margutti ${ }^{1}$, Alicia M. Soderberg ${ }^{1}$, Giuliano Pignata ${ }^{2}$, Laura Chomiuk $^{1,3}$, \\ Robert A. Fesen ${ }^{4}$, Filomena Bufano ${ }^{2}$, Nathan E. Sanders $^{1}$, Jerod T. Parrent ${ }^{4,5}$, Stuart Parker ${ }^{6}$, Padlo Mazzali $^{7}, 8,9$, \\ Elena Pian ${ }^{9}, 10,11$, Timothy Pickering ${ }^{12,13}$, David A. H. Buckley ${ }^{12,13}$, Steven M. Crawford ${ }^{12,13}$, Amanda A. S. Gulbis ${ }^{12,13}$, \\ Christian Hettlage ${ }^{12,13}$, Eric Hooper ${ }^{14}$, Kenneth H. NordsiecK ${ }^{14}$, Darragh O’Donoghue ${ }^{13}$, Tim-Oliver Husser ${ }^{15}$, \\ Stephen Potter $^{12}$, Alexei Kniazev ${ }^{12,13}$, Paul Kotze ${ }^{12,13}$, EnCarni Romero-Colmenero ${ }^{12,13}$, Petri Vaisanen ${ }^{12,13}$, \\ Marsha Wolf ${ }^{14}$, Michael F. Bietenholz ${ }^{16,17}$, Norbert Bartel ${ }^{16}$, Claes Fransson ${ }^{18}$, Emma S. Walker ${ }^{11}$, \\ Andreas Brunthaler ${ }^{3,19}$, Sayan Chakraborti ${ }^{20}$, Emily M. Levesque ${ }^{21}$, Andrew MacFadyen ${ }^{22}$, Colin Drescher ${ }^{23}$, \\ Greg Bock $^{24}$, Peter Marples ${ }^{25}$, Joseph P. Anderson ${ }^{26}$, Stefano Benetti ${ }^{8}$, Daniel Reichart ${ }^{27}$, and Kevin Ivarsen ${ }^{27}$ \\ ${ }^{1}$ Harvard-Smithsonian Center for Astrophysics, 60 Garden Street, Cambridge, MA 02138, USA; dmilisav@cfa.harvard.edu \\ ${ }^{2}$ Departamento de Ciencias Fisicas, Universidad Andres Bello, Avda. Republica 252, Santiago, Chile \\ ${ }^{3}$ National Radio Astronomy Observatory, P.O. Box O, Socorro, NM 87801, USA \\ ${ }^{4}$ Department of Physics \& Astronomy, Dartmouth College, 6127 Wilder Lab, Hanover, NH 03755, USA \\ ${ }^{5}$ Las Cumbres Observatory Global Telescope Network, Goleta, CA 93117, USA \\ ${ }^{6}$ Parkdale Observatory, 225 Warren Road, RDl Oxford, Canterbury 7495, New Zealand \\ ${ }^{7}$ Max-Planck-Institut für Astrophysik, Karl-Schwarzschild-Strasse 1, D-85748 Garching, Germany \\ ${ }^{8}$ INAF-Osservatorio Astronomico di Padova, Vicolo dell'Osservatorio 5, I-35122 Padova, Italy \\ ${ }^{9}$ Kavli Institute for Theoretical Physics, Kohn Hall, University of California at Santa Barbara, Santa Barbara, CA 93106-4030, USA \\ ${ }^{10}$ INAF, Trieste Astronomical Observatory, Via G. B. Tiepolo 11, I-34143 Trieste, Italy \\ ${ }^{11}$ Scuola Normale Superiore, Piazza dei Cavalieri 7, I-56126 Pisa, Italy \\ 12 South African Astronomical Observatory, P.O. Box 9, Observatory 7935, Cape Town, South Africa \\ ${ }^{13}$ Southern African Large Telescope, P.O. Box 9, Observatory 7935, Cape Town, South Africa \\ ${ }^{14}$ Department of Astronomy, University of Wisconsin, Madison, WI 53706, USA \\ 15 Institut für Astrophysik, Georg-August-Universität, Friedrich-Hund-Platz 1, D-37077 Göttingen, Germany \\ ${ }^{16}$ Department of Physics and Astronomy, York University, Toronto, Ontario M3J 1P3, Canada \\ ${ }^{17}$ Hartebeesthoek Radio Observatory, P.O. Box 443, Krugersdorp 1740, South Africa \\ ${ }^{18}$ Department of Astronomy, The Oskar Klein Centre, Stockholm University, SE-10691 Stockholm, Sweden \\ ${ }^{19}$ Max-Planck-Institut für Radioastronomie, Auf dem Hügel 69, D-53121 Bonn, Germany \\ ${ }^{20}$ Department of Astronomy and Astrophysics, Tata Institute of Fundamental Research, 1 Homi Bhabha Road, Mumbai 400005, India \\ ${ }^{21}$ CASA, Department of Astrophysical and Planetary Sciences, University of Colorado, 389-UCB, Boulder, CO 80309, USA \\ ${ }_{22}$ Department of Physics, New York University, 4 Washington Place, New York, NY 10003, USA \\ ${ }_{23}$ PO Box 1555, Sunnybank Hills 4109, Queensland, Australia \\ ${ }^{24}$ Windaroo Lakes Observatory, 2 Anna-Louise Terrace, Windaroo, Queensland, Australia \\ ${ }^{25}$ Loganholme Observatory, 45 Kiewa Drive, Loganholme, Queensland, Australia \\ ${ }^{26}$ Departamento de Astronomia, Universidad de Chile, Casilla 36-D, Santiago, Chile \\ ${ }^{27}$ Department of Physics and Astronomy, University of North Carolina at Chapel Hill, Campus Box 3255, Chapel Hill, NC 27599-3255, USA \\ Received 2012 July 9; accepted 2013 February 7; published 2013 March 26
}

\begin{abstract}
We present X-ray, UV/optical, and radio observations of the stripped-envelope, core-collapse supernova (SN) 2011ei, one of the least luminous SNe IIb or Ib observed to date. Our observations begin with a discovery within $\sim 1$ day of explosion and span several months afterward. Early optical spectra exhibit broad, Type II-like hydrogen Balmer profiles that subside rapidly and are replaced by Type Ib-like He-rich features on a timescale of one week. High-cadence monitoring of this transition suggests absorption attributable to a high-velocity $(\gtrsim 12,000 \mathrm{~km} \mathrm{~s}$ ) $\mathrm{H}$-rich shell, which is likely present in many Type Ib events. Radio observations imply a shock velocity of $v \approx 0.13 c$ and a progenitor star average mass-loss rate of $\dot{M} \approx 1.4 \times 10^{-5} M_{\odot} \mathrm{yr}^{-1}$ (assuming wind velocity $v_{w}=10^{3} \mathrm{~km} \mathrm{~s}^{-1}$ ). This is consistent with independent constraints from deep X-ray observations with Swift-XRT and Chandra. Overall, the multi-wavelength properties of SN 2011ei are consistent with the explosion of a lowermass $\left(3-4 M_{\odot}\right)$, compact $\left(R_{*} \lesssim 1 \times 10^{11} \mathrm{~cm}\right)$, He-core star. The star retained a thin hydrogen envelope at the time of explosion, and was embedded in an inhomogeneous circumstellar wind suggestive of modest episodic mass loss. We conclude that SN 2011ei's rapid spectral metamorphosis is indicative of time-dependent classifications that bias estimates of the relative explosion rates for Type IIb and Ib objects, and that important information about a progenitor star's evolutionary state and mass loss immediately prior to SN explosion can be inferred from timely multi-wavelength observations.
\end{abstract}

Key words: supernovae: general - supernovae: individual (SN 2011ei)

Online-only material: color figures

\section{INTRODUCTION}

Core-collapse supernovae (SNe) are traditionally defined by their spectroscopic properties in the optical (see Filippenko 1997 for review). SNe Type II show hydrogen lines at all epochs. SNe Type $\mathrm{Ib}$ do not show hydrogen lines, but do show conspicuous $\mathrm{He}$ features, and SNe Type Ic show neither $\mathrm{H}$ nor He. These classifications are thought to reflect compositional differences 
in the envelopes of the massive progenitor stars immediately prior to explosion. The envelopes of $\mathrm{SNe} \mathrm{Ib}$ are $\mathrm{H}$-deficient while those of SNe Ic are both $\mathrm{H}$ - and He-deficient.

However, exceptions and transitional subtypes exist that blur these formal classifications. Observations of SN 1987K (Filippenko 1988) showed a gradual transition in its optical spectra from Type II-like lines at photospheric epochs $(\lesssim 2$ months post-outburst) to Type Ib-like features at nebular epochs $(\gtrsim 5$ months). This remarkable spectroscopic evolution demonstrated the existence of an intermediary between the H-rich Type II and H-deficient Type Ib and Ic classes (hereafter Type Ibc). SN 1993J (Filippenko et al. 1993) was a well-studied additional example of this breaching of SN classifications and has come to be thought of as a prototype of SNe Type IIb (Woosley et al. 1994). Like the $\mathrm{SNe}$ Ibc objects, $\mathrm{SNe}$ IIb are believed to be massive stars but only partially stripped of their outer H-rich envelopes.

The entire class of SNe IIb and Ibc are collectively known as "stripped-envelope" events (Clocchiatti et al. 1997). The degree of $\mathrm{H}$ and He envelope deficiency is believed to be the consequence of varying degrees of an SN progenitor star's mass loss. Potential mechanisms for mass loss include steady winds (Puls et al. 2008), eruptive mass-loss episodes (Smith \& Owocki 2006), or mass transfer due to Roche lobe overflow in a close binary system (Podsiadlowski et al. 1992). It is an open issue which of these processes dominate, and whether they occur in relatively high-mass single-star progenitors $\left(M \gtrsim 20 M_{\odot}\right)$, in lower-mass progenitors $\left(M \gtrsim 10 M_{\odot}\right)$, or a mixture of both.

The wide diversity in the small number of well-observed objects has complicated attempts at establishing firm connections between SN subtypes and their progenitor systems. This has been particularly the case for the inferred progenitors of SNe IIb, which have been quite varied among a few nearby cases. For example, late-time spectroscopic and photometric observations of SN 1993J showed compelling evidence for a massive binary companion to the red supergiant progenitor star (Aldering et al. 1994; Maund et al. 2004). However, pre-explosion imaging with the Hubble Space Telescope of SN 2008ax suggests that it most likely came from a more compact progenitor, such as a single massive Wolf-Rayet (W-R) star or an interacting binary in a low-mass cluster (Crockett et al. 2008). Most recently, there has been considerable discourse about a yellow supergiant discovered in pre-explosion imaging at the location of SN 2011dh and its viability as a candidate progenitor (Maund et al. 2011; Van Dyk et al. 2011; Arcavi et al. 2011; Soderberg et al. 2012; Murphy et al. 2011; Bietenholz et al. 2012; Georgy 2012).

From a statistical standpoint, the relatively high frequency of stripped-envelope $\mathrm{SNe}(\sim 30 \%$ of all core-collapse events by volume; Li et al. 2011) in combination with many nondetections of progenitor stars in pre-explosion imaging (Smartt 2009) argues that at least some progenitor scenarios must involve massive stars in binary systems (Podsiadlowski et al. 1992; Fryer et al. 2007; Eldridge et al. 2008; Smith et al. 2011). Observational and theoretical investigations have suggested binary mass-loss scenarios that could lead to a spectroscopic sequence of SN types from IIb $\rightarrow \mathrm{Ib} \rightarrow$ Ic (e.g., Filippenko et al. 1994; Woosley et al. 1994; Nomoto et al. 1995; Heger et al. 2003; Yoon et al. 2010; Dessart et al. 2011). Chevalier \& Soderberg (2010) point out that the sequence may only be applicable for a subset of this population in light of optical and radio data that favor a division among $\mathrm{SNe}$ IIb progenitors between objects like SN 1993J that have extended envelopes $\left(R_{*} \sim 10^{13} \mathrm{~cm}\right.$; referred to as Type "eIIb") and others like SN 2008ax that are more compact ( $R_{*} \sim 10^{11} \mathrm{~cm}$; Type "cIlb"). They argue that Type cIIb closely resemble Type Ib, and that the two subtypes may be related by a gradual transition depending on remaining $\mathrm{H}$ mass.

One key consequence of the proposed stripped-envelope sequence is that a thin hydrogen layer is expected to be present in many SNe Ib progenitors at the pre-SN stage, and it is theorized that it may be possible to see hydrogen in their early spectra. Thus, the detection of hydrogen absorption lines at high velocity in $\mathrm{SNe} \mathrm{Ib}$ is important because of its implications for the nature and evolutionary stage of the progenitor stars.

However, the existence of hydrogen in $\mathrm{SNe} \mathrm{Ib}$ remains somewhat uncertain. Reports of suspected high-velocity $\left(\gtrsim 12,000 \mathrm{~km} \mathrm{~s}^{-1}\right.$ ) hydrogen absorption around $6250 \AA$ exist; e.g., SN 1999dn, SN 2000H (Branch et al. 2002), SN 2005bf (Anupama et al. 2005; Folatelli et al. 2006; Parrent et al. 2007), and SN 2008D (Soderberg et al. 2008). Alternative line identifications, however, have been offered. For instance, C II $\lambda 6580$, being less than $800 \mathrm{~km} \mathrm{~s}^{-1}$ to the red of $\mathrm{H} \alpha$, has been considered a plausible identification (e.g., Harkness et al. 1987; Deng et al. 2000), and Si II $\lambda 6355$ and $\mathrm{Ne}$ I $\lambda 6402$ have also been suggested (Hamuy et al. 2002; Tanaka et al. 2009; Branch 1972; Benetti et al. 2002).

The uncertain identification of hydrogen in $\mathrm{SNe} \mathrm{Ib}$ may be a consequence of current limitations in spectroscopic followup of SN candidates. Because spectra of SNe shortly after outburst are relatively rare, there is the possibility that $\mathrm{SNe} \mathrm{IIb}$ versus Ib (versus Ic?) classification may be biased by the time of observation (see discussions by Branch et al. 2002 and Chornock et al. 2011). Indeed, precise rates of these events may be compromised by the dearth of early-time spectroscopic and photometric observations several days prior to maximum light when the $\mathrm{SN}$ is faint and the photosphere still resides in the outer layers of the progenitor star. Almost half of the ejecta mass may have passed through the photosphere by the time peak brightness is reached, thus observations within a few days of explosion offer useful constraints on the progenitor star's current evolutionary state (Dessart et al. 2011; Yoon et al. 2010).

Our discovery and subsequent monitoring of SN 2011ei is an example of the utility of very early data. Discovered in NGC 6925 on July 25.434 UT (all future dates also in UT) as part of the Backyard Observatory Supernova Search (BOSS), early optical spectra of SN 2011ei initially showed line features similar to those of the Type IIb SN 1993J and SN 1996cb, but with a noticeably broader $\mathrm{H} \alpha$ P-Cygni profile reminiscent of the energetic Type IIb SN 2003bg (Stritzinger et al. 2011; Milisavljevic et al. 2011). However, spectra taken approximately one week later showed rapid evolution away from strong hydrogen features to conspicuous Type Ib-like helium absorptions.

This rapid change in SN 2011ei's spectral properties along with a contemporaneous radio detection motivated a coordinated, multi-wavelength monitoring program presented here. In Sections 2-5, we present and analyze X-ray, ultraviolet (UV), optical, and radio observations from Swift, Chandra, and ground-based observatories including the Southern African Large Telescope (SALT), Magellan, and the Karl G. Jansky Very Large Array (VLA). In Section 6, we discuss the implications these findings have for SN 2011ei's progenitor star and its massloss history. Finally, in Section 7, we summarize our findings and discuss their relevance to a wider subset of stripped-envelope core-collapse $\mathrm{SNe}$. 


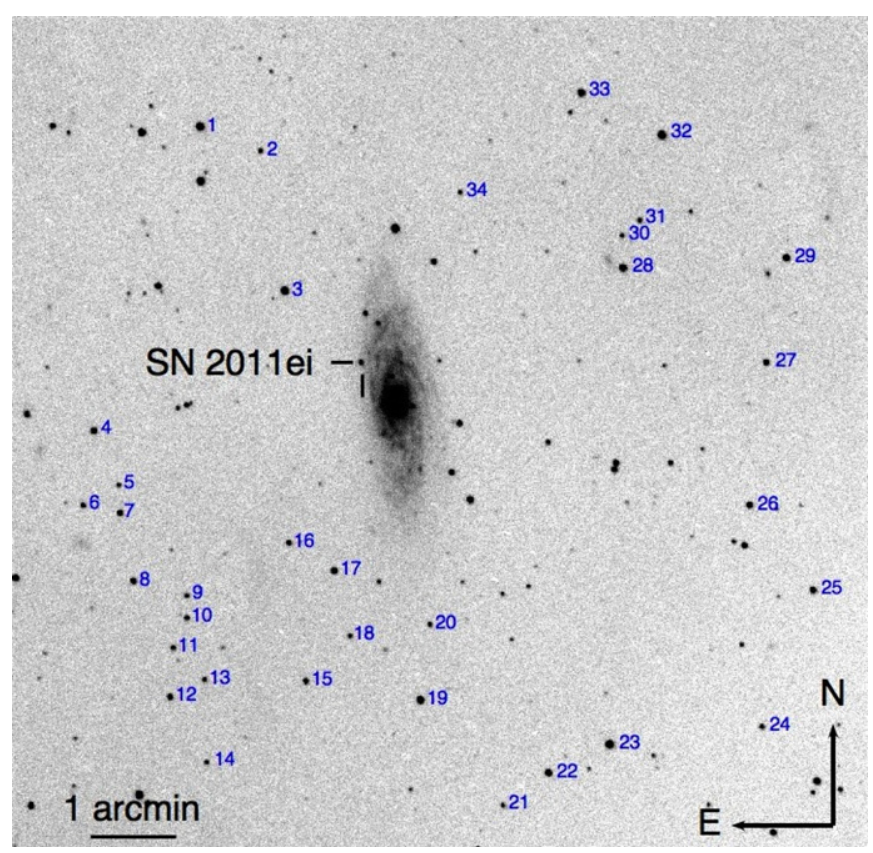

Figure 1. PROMPT image of SN 2011ei around the time of maximum light and its host galaxy NGC 6925. Local sequence stars used to calibrate stars are numbered according to Table 2 .

(A color version of this figure is available in the online journal.)

\section{OBSERVATIONS}

\subsection{Distance and Reddening}

Located at coordinates $\alpha=20^{\mathrm{h}} 34^{\mathrm{m}} 22^{\mathrm{s}} .62$ and $\delta=$ $-31^{\circ} 58^{\prime} 23^{\prime \prime} 6$ (J2000.0), SN 2011ei is situated in projection along the outer periphery of its host spiral galaxy NGC 6925 (see Figure 1). Estimates of the distance to NGC 6925 via Tully-Fisher measurements range between $23.3 \mathrm{Mpc}$ and $35.2 \mathrm{Mpc}$ (Willick et al. 1997). For all calculations we adopt a distance $D=28.5 \pm 5.7 \mathrm{Mpc}$ and a distance modulus $\mu=32.27 \pm 0.43$ (Springob et al. 2009).

The reddening due to the Milky Way in the direction of NGC 6925 is $E(B-V)_{m w}=0.059$ mag according to the infrared dust maps of Schlegel et al. (1998). NGC 6925 is highly inclined which would suggest possibly significant local extinction along its disk plane. The SN, however, appears to lie on the galaxy's near side. We used the empirically derived relation between equivalent width (EW) of $\mathrm{Na}$ I $\mathrm{D}$ absorption and $E(B-V)$ described in Turatto et al. (2003) to estimate the host extinction (though for a discussion of possible problems with this relationship, see, e.g., Poznanski et al. 2011). The $\mathrm{EW}(\mathrm{Na}$ I D) $=1.14 \pm 0.10 \AA$ absorption at the redshift of NGC 6925 determined from the weighted average of five spectra suggests a host extinction of $E(B-V)_{\text {host }}=0.18 \mathrm{mag}$. Combining the inferred host and Galactic extinction, we adopt a total reddening $E(B-V)=0.24 \mathrm{mag}$ and assume $R_{V}=$ $A_{V} / E(B-V)=3$.1. In Section 3, we show that this estimate of the reddening is likely an upper limit.

\subsection{X-Ray Observations with Swift-XRT and Chandra}

We promptly requested X-ray observations from the Swift spacecraft (Gehrels et al. 2004) using the XRT instrument (Burrows et al. 2005) beginning 2011 August 3.2. The total exposure time was $54 \mathrm{ks}$. Data were analyzed using the latest version of the HEASOFT package available at the time of writing (version 6.11) and corresponding calibration files. Standard filtering and screening criteria were applied. No X-ray source is detected coincident with SN 2011ei with a $3 \sigma$ upper limit of $4 \times 10^{-3}$ counts s$^{-1}$ in the $0.3-10 \mathrm{keV}$ band. The Galactic neutral hydrogen column density in the direction of SN 2011ei is $4.76 \times 10^{20} \mathrm{~cm}^{-2}$ (Kalberla et al. 2005). Assuming a spectral photon index $\Gamma=2$, this upper limit translates to an absorbed (unabsorbed) flux of $1.4 \times 10^{-13} \mathrm{erg} \mathrm{cm}^{-2} \mathrm{~s}^{-1}$ $\left(1.6 \times 10^{-13} \mathrm{erg} \mathrm{cm}^{-2} \mathrm{~s}^{-1}\right)$, corresponding to a luminosity of $1.5 \times 10^{40} \mathrm{erg} \mathrm{s}^{-1}$.

From the Swift-XRT images, X-ray contamination from the host galaxy nucleus is clearly identified at the SN site. Consequently, a 10 ks Chandra X-ray Observatory observation was also obtained on 2011 August 21 to resolve SN emission from the host galaxy nucleus (PI: Soderberg; Prop. 12500613). Data were reduced with the CIAO software package (version 4.3) with calibration database CALDB (version 4.4.2). Standard filtering using CIAO threads for ACIS data was applied.

Consistent with the Swift-XRT observation, no X-ray source is detected at the SN position in the Chandra observation with a $3 \sigma$ upper limit of $7.6 \times 10^{-4}$ counts s$^{-1}$ in the $0.5-8 \mathrm{keV}$ band. From the count rate, we derived an absorbed flux limit of $5.5 \times 10^{-15} \mathrm{erg} \mathrm{s}^{-1} \mathrm{~cm}^{-2}$. Using the Galactic relations between $N_{\mathrm{H}}$ and $A_{V}$ (Predehl \& Schmitt 1995; Watson 2011), an intrinsic hydrogen column density $N_{\mathrm{H}[\mathrm{int}]} \sim 1.5 \times 10^{21} \mathrm{~cm}^{-2}$ is inferred. Accounting for the total absorption (Galactic plus intrinsic) leads to an unabsorbed flux limit of $7 \times 10^{-15} \mathrm{erg} \mathrm{s}^{-1} \mathrm{~cm}^{-2}$ (assuming a simple power-law model with spectral photon index $\Gamma=2$ ), corresponding to an X-ray luminosity of $L_{X[0.5-8 \mathrm{keV}]}<7 \times 10^{38} \mathrm{erg} \mathrm{s}^{-1}$. By comparison, X-ray emission at similar epochs from an SN IIb such as SN 1993J would have been detected $\left(L_{X[0.3-8 \mathrm{keV}]} \approx 8 \times 10^{39} \mathrm{erg} \mathrm{s}^{-1}\right)$, whereas the emission from an object like SN 2011dh $\left(L_{X[0.3-8 \mathrm{keV}]} \approx\right.$ $1.5 \times 10^{38} \mathrm{erg} \mathrm{s}^{-1}$ ) would not have been detected (Soderberg et al. 2012).

\subsection{Ultraviolet and Optical Photometry}

UV/optical observations of SN 2011ei with the Swift-UVOT instrument (Roming et al. 2005) began 2011 August 3.2 and continued through to 2011 September 10.9. Data were acquired using all of its six broadband filters that span the wavelength range of approximately 1600-6000 $\mathrm{A}$. The Swift-UVOT photometry presented in Table 1 is based on the photometric system described in Poole et al. (2008). In this system the Swift $b$ and $v$ filters are roughly equivalent to the standard Johnson-Kron-Cousins $B$ and $V$ filters (see Poole et al. 2008 for details).

We analyzed the Swift-UVOT photometric data following the prescriptions of Brown et al. (2009). A 3" aperture was used to maximize the signal-to-noise ratio $(\mathrm{S} / \mathrm{N})$. Late-time images acquired 2012 March 27-April 6 have been used to estimate and remove the underlying host galaxy contribution. Observations beyond 2011 September 08 are not reported because SN emission was too faint to obtain reliable measurements. Also not reported are observations in the $u v w 2$ and $u v m 2$ filters where the $\mathrm{SN}$ was not detected with significant $\mathrm{S} / \mathrm{N}$.

Also reported in Table 1 is $B V R I$ photometry acquired with the Panchromatic Robotic Optical Monitoring and Polarimetry Telescope (PROMPT; Reichart et al. 2005) PROMPT3 and PROMPT5 located at Cerro Tololo Inter-American Observatory. All images were dark subtracted and flat-field corrected. Frames taken with the same filter were stacked in order to produce a final deeper image. The instrumental magnitude of the SN 
Table 1

Combined Swift-UVOT and PROMPT Photometry of SN 2011ei

\begin{tabular}{|c|c|c|c|c|c|c|c|c|}
\hline \multirow{2}{*}{$\begin{array}{l}\text { JD } \\
-2400000\end{array}$} & \multicolumn{4}{|c|}{ Swift-UVOT } & \multicolumn{4}{|c|}{ PROMPT } \\
\hline & $u v w 1$ & $u$ & $b$ & $v$ & $B$ & $V$ & $R$ & $I$ \\
\hline 55769.73 & $\ldots$ & $\ldots$ & $\ldots$ & $\ldots$ & 19.2640 .058 & 18.7860 .104 & $\ldots$ & $\ldots$ \\
\hline 55770.65 & $\ldots$ & $\ldots$ & $\ldots$ & $\ldots$ & $\ldots$ & 18.4450 .065 & 18.0870 .024 & 18.1270 .049 \\
\hline 55776.82 & $\ldots$ & 16.670 .06 & 17.630 .07 & 17.580 .11 & $\ldots$ & $\ldots$ & $\ldots$ & $\ldots$ \\
\hline 55776.84 & 17.950 .07 & $\ldots$ & $\ldots$ & $\ldots$ & $\ldots$ & $\ldots$ & $\ldots$ & $\ldots$ \\
\hline 55777.71 & 18.310 .12 & 16.750 .09 & 17.860 .11 & $\ldots$ & $\ldots$ & $\ldots$ & $\ldots$ & $\ldots$ \\
\hline 55778.52 & 17.900 .13 & $\ldots$ & $\ldots$ & 17.530 .21 & $\ldots$ & $\ldots$ & $\ldots$ & $\ldots$ \\
\hline 55779.25 & $\ldots$ & 16.670 .07 & 17.440 .08 & $\ldots$ & $\ldots$ & $\ldots$ & $\ldots$ & $\ldots$ \\
\hline 55779.32 & 17.820 .09 & $\ldots$ & $\ldots$ & 17.410 .15 & $\ldots$ & $\ldots$ & $\ldots$ & $\ldots$ \\
\hline 55780.64 & 17.910 .10 & 16.610 .08 & 17.380 .08 & $\begin{array}{lll}17.31 & 0.14\end{array}$ & $\ldots$ & $\ldots$ & $\ldots$ & $\ldots$ \\
\hline 55782.61 & 17.900 .10 & $\ldots$ & $\ldots$ & 17.100 .12 & $\ldots$ & $\ldots$ & $\ldots$ & $\ldots$ \\
\hline 55783.14 & $\ldots$ & 16.700 .07 & 17.380 .08 & $\ldots$ & $\ldots$ & $\ldots$ & $\ldots$ & $\ldots$ \\
\hline 55783.75 & 18.040 .16 & $\ldots$ & $\ldots$ & $\ldots$ & $\ldots$ & $\ldots$ & $\ldots$ & $\ldots$ \\
\hline 55783.77 & $\ldots$ & $\ldots$ & $\ldots$ & $\ldots$ & 17.3810 .020 & 17.0310 .013 & 16.8660 .011 & 16.7290 .019 \\
\hline 55784.68 & $\ldots$ & $\ldots$ & $\ldots$ & $\ldots$ & 17.3620 .017 & 17.0200 .017 & 16.8460 .012 & 16.6950 .021 \\
\hline 55785.58 & $\ldots$ & $\ldots$ & $\ldots$ & $\ldots$ & 17.4220 .019 & 17.0060 .025 & 16.8130 .022 & $\ldots$ \\
\hline 55788.13 & 18.310 .11 & 17.270 .10 & 17.480 .09 & 16.990 .11 & $\ldots$ & $\ldots$ & $\ldots$ & $\ldots$ \\
\hline 55788.70 & $\ldots$ & $\ldots$ & $\ldots$ & $\ldots$ & 17.6360 .018 & 17.0060 .019 & 16.7960 .013 & 16.5750 .021 \\
\hline 55788.96 & 18.400 .12 & 17.440 .11 & 17.480 .09 & $\ldots$ & $\ldots$ & $\ldots$ & $\ldots$ & $\ldots$ \\
\hline 55789.30 & $\ldots$ & $\ldots$ & $\ldots$ & 17.070 .10 & $\ldots$ & $\ldots$ & $\ldots$ & $\ldots$ \\
\hline 55789.66 & 18.560 .13 & 17.550 .12 & 17.490 .09 & $\ldots$ & $\ldots$ & $\ldots$ & $\ldots$ & $\ldots$ \\
\hline 55791.03 & 18.490 .13 & 17.640 .13 & 17.610 .09 & $\ldots$ & $\ldots$ & $\ldots$ & $\ldots$ & $\ldots$ \\
\hline 55791.44 & $\ldots$ & $\ldots$ & $\ldots$ & 17.170 .11 & $\ldots$ & $\ldots$ & $\ldots$ & $\ldots$ \\
\hline 55792.04 & 18.430 .12 & $\ldots$ & $\ldots$ & $\ldots$ & $\ldots$ & $\ldots$ & $\ldots$ & $\ldots$ \\
\hline 55792.23 & $\ldots$ & 18.120 .15 & 17.840 .10 & $\ldots$ & $\ldots$ & $\ldots$ & $\ldots$ & $\ldots$ \\
\hline 55792.63 & 18.740 .23 & $\ldots$ & $\ldots$ & $\ldots$ & $\ldots$ & $\ldots$ & $\ldots$ & $\ldots$ \\
\hline 55794.01 & 18.610 .18 & $\ldots$ & $\ldots$ & $\ldots$ & $\ldots$ & $\ldots$ & $\ldots$ & $\ldots$ \\
\hline 55794.48 & $\ldots$ & 18.700 .23 & 18.150 .12 & $\ldots$ & $\ldots$ & $\ldots$ & $\ldots$ & $\ldots$ \\
\hline 55794.59 & $\ldots$ & $\ldots$ & $\ldots$ & $\ldots$ & 18.3450 .031 & 17.3540 .019 & 16.9500 .019 & 16.6260 .026 \\
\hline 55795.18 & 18.930 .16 & $\ldots$ & $\ldots$ & $\ldots$ & $\ldots$ & $\ldots$ & $\ldots$ & $\ldots$ \\
\hline 55795.73 & $\ldots$ & $\ldots$ & $\ldots$ & $\ldots$ & 18.5080 .038 & 17.4970 .027 & 16.9990 .018 & 16.6740 .022 \\
\hline 55796.13 & $\ldots$ & 19.120 .29 & 18.410 .15 & $\ldots$ & $\ldots$ & $\ldots$ & $\ldots$ & $\ldots$ \\
\hline 55796.59 & 18.940 .12 & $\ldots$ & $\ldots$ & $\ldots$ & $\ldots$ & $\ldots$ & $\ldots$ & $\ldots$ \\
\hline 55798.09 & $\ldots$ & $\ldots$ & 18.610 .16 & $\ldots$ & $\ldots$ & $\ldots$ & $\ldots$ & $\ldots$ \\
\hline 55798.72 & $\ldots$ & $\ldots$ & $\ldots$ & $\ldots$ & 18.9150 .054 & 17.7210 .022 & 17.0850 .015 & 16.7650 .019 \\
\hline 55800.13 & 18.950 .12 & 19.590 .43 & $\ldots$ & $\ldots$ & $\ldots$ & $\ldots$ & $\ldots$ & $\ldots$ \\
\hline 55802.11 & $\ldots$ & $\ldots$ & 18.920 .19 & $\ldots$ & $\ldots$ & $\ldots$ & $\ldots$ & $\ldots$ \\
\hline 55802.74 & $\ldots$ & $\ldots$ & $\ldots$ & $\ldots$ & 19.1310 .242 & 17.9800 .034 & 17.2100 .017 & 16.8460 .018 \\
\hline 55804.04 & 19.040 .12 & 20.050 .63 & $\ldots$ & $\ldots$ & $\ldots$ & $\ldots$ & $\ldots$ & $\ldots$ \\
\hline 55805.77 & $\ldots$ & $\ldots$ & $\ldots$ & $\ldots$ & $\ldots$ & 18.1850 .023 & 17.2970 .017 & 16.9730 .017 \\
\hline 55805.98 & $\ldots$ & $\ldots$ & 19.270 .25 & $\ldots$ & $\ldots$ & $\ldots$ & $\ldots$ & $\ldots$ \\
\hline 55808.15 & $\ldots$ & 19.980 .67 & $\ldots$ & $\ldots$ & $\ldots$ & $\ldots$ & $\ldots$ & $\ldots$ \\
\hline 55819.61 & $\ldots$ & $\ldots$ & $\ldots$ & $\ldots$ & $\ldots$ & 18.3370 .055 & 17.5520 .020 & 17.1670 .024 \\
\hline
\end{tabular}

Note. Uncertainties are adjacent to measurements and are at the $68 \%$ confidence level.

was measured in all the stacked frames using the point-spread function technique after a scaled template image was subtracted from the SN image. SN photometry was calibrated against a sequence of stars located close to the SN. This local sequence, with magnitudes reported in Table 2, was calibrated to the standard Johnson-Kron-Cousins photometric systems using observations of Landolt $(1992,2007)$ standard stars.

In addition to Swift-UVOT and PROMPT photometry, we estimate an apparent magnitude $R=18.0 \pm 0.4$ on 2011 July 25.434 (JD 2455767.93) based on unfiltered discovery images of the SN. The data were obtained with the $0.35 \mathrm{~m}$ Celestron telescope equipped with a KAF-3200ME camera most sensitive to $R$-band emission at Parkdale Observatory, Canterbury, New Zealand. After flat fielding and correcting for the bias and dark current levels, SN emission was calibrated against field comparison stars of the USNO-B 1.0 star catalog and then converted to the standard $R$ value.

\subsection{Optical Spectroscopy}

A total of 16 epochs of long-slit optical spectra of SN 2011ei were collected. The details of all observations are provided in Table 3 and the reduced spectra are plotted in Figure 2. Most observations were made at SAAO Observatory with the $10 \mathrm{~m}$ SALT using the Robert Stobie Spectrograph (RSS; Burgh et al. 2003). The holographic grism pg0900 was used at combinations of four tilts to span a wavelength window of 3200-9000 $\AA$ and cover gaps between CCD detectors. On chip CCD $4 \times 4$ binning in the spectral and spatial directions was employed, yielding a dispersion of $1.96 \AA$ pixel $^{-1}$ and $\approx 6 \AA$ FWHM resolution. An atmospheric dispersion corrector minimized the effects of atmospheric refraction for all observations which were made generally around an airmass of 1.2. Conditions were mostly non-photometric with a median seeing around 1".3.

Additional spectra were obtained using a variety of telescopes and instrumental setups. The Magellan $6.5 \mathrm{~m}$ Baade and Clay 
Table 2

PROMPT Photometry of the Local Sequence Stars in the Field of NGC 6925

\begin{tabular}{|c|c|c|c|c|c|c|}
\hline No. & R.A. (J2000.0) & Decl. (J2000.0) & $B$ & V & $R$ & $I$ \\
\hline 1 & $20: 34: 31.425$ & $-31: 55: 37.13$ & 15.3500 .023 & 14.6580 .033 & 14.2680 .036 & 13.8980 .081 \\
\hline 2 & $20: 34: 28.104$ & $-31: 55: 54.40$ & 17.9040 .031 & 17.1830 .027 & 16.7580 .025 & 16.3800 .025 \\
\hline 3 & $20: 34: 26.771$ & $-31: 57: 32.56$ & 15.6800 .015 & 14.9890 .027 & 14.5740 .037 & 14.2200 .048 \\
\hline 4 & $20: 34: 37.298$ & $-31: 59: 10.83$ & 16.8430 .012 & 16.1050 .034 & 15.6660 .044 & 15.2350 .048 \\
\hline 5 & $20: 34: 35.922$ & $-31: 59: 48.73$ & 17.7460 .023 & 17.3350 .031 & 17.0270 .045 & 16.7240 .046 \\
\hline 6 & $20: 34: 37.883$ & $-32: 00: 03.00$ & 17.7210 .028 & 17.0530 .031 & 16.6600 .017 & 16.2830 .029 \\
\hline 7 & $20: 34: 35.853$ & $-32: 00: 08.29$ & 17.1390 .057 & 16.4640 .039 & 16.0410 .028 & 15.6820 .062 \\
\hline 8 & $20: 34: 35.118$ & $-32: 00: 55.90$ & 17.3490 .029 & 16.4110 .024 & 15.8580 .048 & 15.4040 .064 \\
\hline 9 & $20: 34: 32.157$ & $-32: 01: 06.60$ & 17.9270 .011 & 17.3960 .035 & 17.0650 .033 & 16.7200 .046 \\
\hline 10 & $20: 34: 32.166$ & $-32: 01: 22.16$ & 17.6580 .062 & 17.0610 .052 & 16.6900 .040 & 16.3440 .046 \\
\hline 11 & $20: 34: 32.923$ & $-32: 01: 43.05$ & 18.4440 .020 & 17.0610 .018 & 16.1290 .046 & 15.3590 .078 \\
\hline 12 & $20: 34: 33.090$ & $-32: 02: 17.52$ & 17.2000 .054 & 16.3630 .034 & 15.8770 .045 & 15.4290 .063 \\
\hline 13 & $20: 34: 31.201$ & $-32: 02: 05.58$ & 18.5350 .158 & 17.2780 .037 & 16.3830 .062 & 15.7220 .063 \\
\hline 14 & $20: 34: 31.086$ & $-32: 03: 03.46$ & 18.0730 .053 & 17.4830 .039 & 17.1050 .054 & 16.7380 .024 \\
\hline 15 & $20: 34: 25.617$ & $-32: 02: 06.45$ & 17.3240 .052 & 16.6610 .033 & 16.2490 .049 & 15.9020 .072 \\
\hline 16 & $20: 34: 26.544$ & $-32: 00: 29.45$ & 17.5760 .026 & 16.7490 .036 & 16.2640 .046 & 15.8350 .073 \\
\hline 17 & $20: 34: 24.061$ & $-32: 00: 48.81$ & 16.6660 .045 & 15.9240 .044 & 15.4770 .046 & 15.0940 .064 \\
\hline 18 & $20: 34: 23.184$ & $-32: 01: 34.76$ & 18.2840 .047 & 17.2220 .044 & 16.5900 .033 & 16.1100 .072 \\
\hline 19 & 20:34:19.306 & $-32: 02: 19.59$ & 15.9620 .029 & 15.2720 .038 & 14.8520 .050 & 14.4920 .070 \\
\hline 20 & $20: 34: 18.786$ & $-32: 01: 26.81$ & 18.2550 .012 & 17.3360 .064 & 16.7650 .069 & 16.3430 .033 \\
\hline 21 & $20: 34: 14.755$ & $-32: 03: 33.72$ & 18.7841 .180 & 17.3780 .044 & 16.9670 .047 & 16.6210 .085 \\
\hline 22 & $20: 34: 12.242$ & $-32: 03: 10.87$ & 16.7580 .043 & 15.7890 .024 & 15.2270 .051 & 14.7580 .075 \\
\hline 23 & $20: 34: 08.874$ & $-32: 02: 50.79$ & 15.7870 .016 & 14.6580 .029 & 13.9200 .051 & 13.3720 .080 \\
\hline 24 & $20: 34: 00.474$ & $-32: 02: 38.43$ & 17.7070 .053 & 16.8330 .041 & 16.3070 .035 & 15.8730 .079 \\
\hline 25 & $20: 33: 57.672$ & $-32: 01: 02.46$ & 16.6100 .053 & 15.8410 .033 & 15.3670 .051 & 14.9790 .088 \\
\hline 26 & 20:34:01.183 & $-32: 00: 02.73$ & 16.5350 .057 & 15.9620 .035 & 15.5930 .046 & 15.2640 .070 \\
\hline 27 & $20: 34: 00.268$ & $-31: 58: 22.97$ & 17.0990 .028 & 16.4280 .030 & 16.0140 .051 & 15.6480 .062 \\
\hline 28 & 20:34:08.158 & $-31: 57: 16.48$ & 16.7100 .011 & 15.2130 .029 & 14.2290 .049 & 13.1770 .067 \\
\hline 29 & $20: 33: 59.155$ & $-31: 57: 09.43$ & 16.8860 .037 & 15.6620 .026 & 14.8420 .022 & 14.2740 .077 \\
\hline 30 & 20:34:08.192 & $-31: 56: 53.97$ & 17.8990 .011 & 17.3000 .013 & 16.8810 .010 & 16.5490 .054 \\
\hline 31 & 20:34:07.231 & $-31: 56: 43.23$ & 17.7370 .026 & 17.1250 .043 & 16.7450 .036 & 16.3820 .052 \\
\hline 32 & 20:34:06.013 & $-31: 55: 43.34$ & 15.4500 .034 & 14.7180 .032 & 14.2960 .043 & 13.9180 .080 \\
\hline 33 & $20: 34: 10.459$ & $-31: 55: 13.60$ & 15.8720 .028 & 15.2440 .045 & 14.8780 .044 & 14.5080 .062 \\
\hline 34 & $20: 34: 17.120$ & $-31: 56: 23.58$ & 17.9160 .102 & 17.4130 .060 & 17.0270 .051 & 16.6780 .035 \\
\hline
\end{tabular}

Note. Uncertainties are adjacent to measurements and are at the $68 \%$ confidence level.

Table 3

Summary of Optical Spectroscopic Observations of SN 2011ei

\begin{tabular}{|c|c|c|c|c|c|c|}
\hline $\begin{array}{l}\text { Date } \\
\text { (UT) }\end{array}$ & $\begin{array}{c}\text { JD } \\
-2400000\end{array}$ & $\begin{array}{l}\text { Phase }^{\mathrm{a}} \\
\text { (days) }\end{array}$ & $\begin{array}{c}\text { Wavelength Range } \\
(\AA)\end{array}$ & $\begin{array}{c}\text { Resolution } \\
(\AA)\end{array}$ & $\begin{array}{l}\text { Telescope/ } \\
\text { Instrument }\end{array}$ & $\begin{array}{c}\text { Exposure } \\
\text { (s) }\end{array}$ \\
\hline 2011 Jul 29 & 55772.32 & -14 & $3200-9000$ & 6 & SALT/RSS & $2 \times 300$ \\
\hline 2011 Aug 2 & 55776.33 & -10 & $3200-9000$ & 6 & SALT/RSS & $2 \times 300$ \\
\hline 2011 Aug 6 & 55780.31 & -6 & $3200-9000$ & 6 & SALT/RSS & $2 \times 600$ \\
\hline 2011 Aug 8 & 55782.30 & -4 & $3400-8800$ & 6 & SALT/RSS & $1 \times 600$ \\
\hline 2011 Aug 9 & 55783.30 & -3 & $5900-9000$ & 6 & SALT/RSS & $1 \times 600$ \\
\hline 2011 Aug 16 & 55789.54 & 3 & $3400-9000$ & 6 & SALT/RSS & $2 \times 600$ \\
\hline 2011 Aug 21 & 55794.62 & 8 & $3600-8900$ & 13 & SOAR/Goodman & $3 \times 450$ \\
\hline 2011 Aug 26 & 55799.50 & 13 & $3400-9000$ & 6 & SALT/RSS & $1 \times 600$ \\
\hline 2011 Aug 29 & 55803.48 & 17 & $3400-9000$ & 6 & SALT/RSS & $1 \times 600$ \\
\hline 2011 Sep 20 & 55824.43 & 38 & $3500-9500$ & 4 & Magellan/IMACS & $1 \times 900$ \\
\hline 2011 Sep 30 & 55834.63 & 48 & $3800-9400$ & 6 & Magellan/LDSS3 & $3 \times 900$ \\
\hline 2011 Oct 18 & 55852.55 & 66 & $3700-9200$ & 18 & NTT/EFOSC2 & $2 \times 1800$ \\
\hline 2011 Oct 24 & 55859.34 & 73 & $3400-8800$ & 6 & SALT/RSS & $1 \times 600$ \\
\hline 2011 Nov 16 & 55881.55 & 95 & $3800-8900$ & 13 & SOAR/Goodman & $2 \times 2700$ \\
\hline 2011 Nov 18 & 55883.55 & 97 & $3500-9500$ & 4 & Magellan/IMACS & $1 \times 1200$ \\
\hline 2012 Jun 18 & 56096.33 & 310 & $3500-9600$ & 14 & VLT/FORS2 & $2 \times 1800$ \\
\hline
\end{tabular}

Notes.

${ }^{\text {a }}$ Phase is with respect to $V$-band maximum on JD 2455786.5 (2011 Aug 13.0).

${ }^{\mathrm{b}}$ FWHM of night-sky emission lines. 


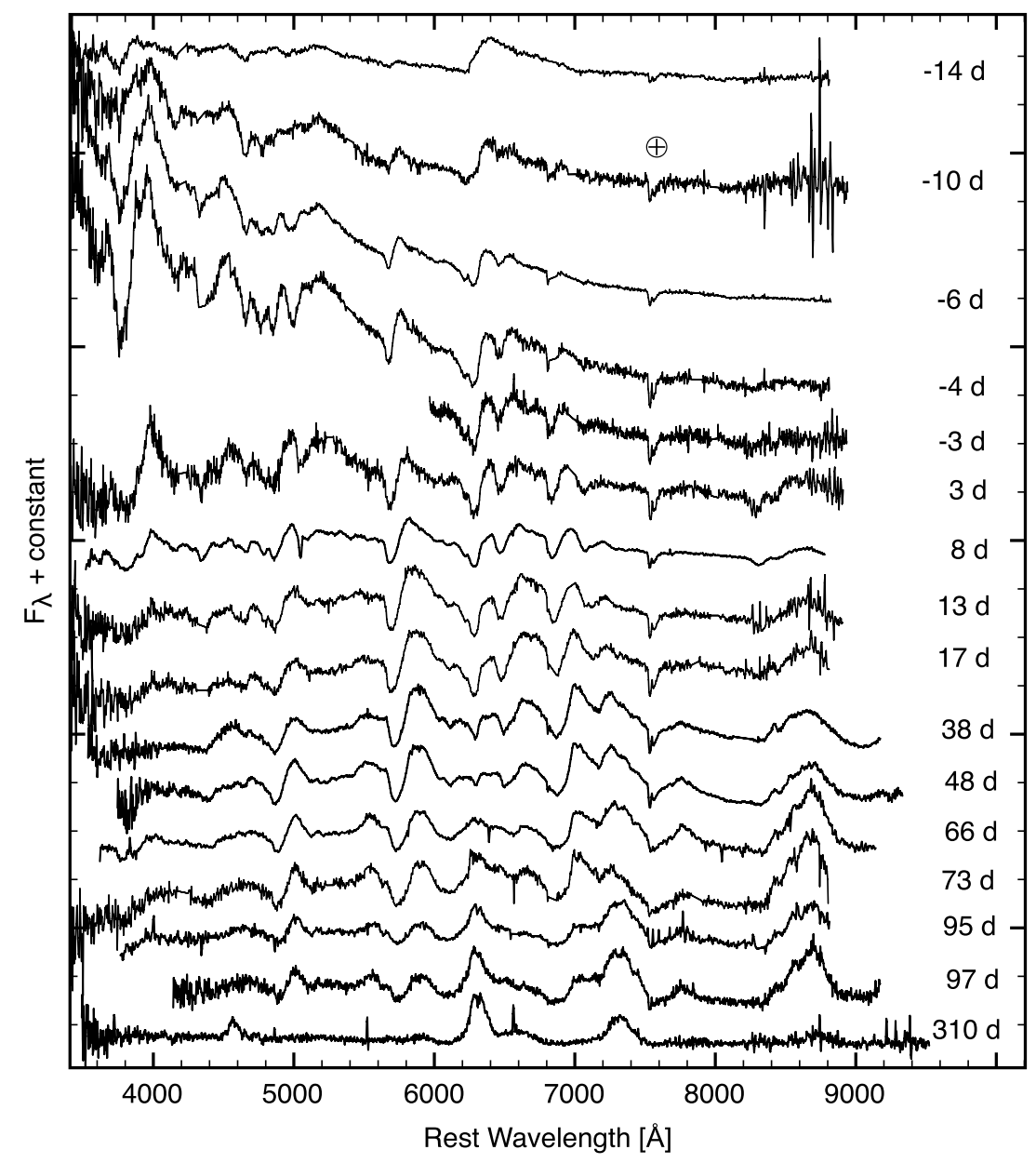

Figure 2. Optical spectra of SN 2011ei. Phase is with respect to $V$-band maximum. Telluric features have been marked with the " $\oplus$ " symbol.

telescopes located at Las Campanas Observatory were used with the IMACS (Bigelow et al. 1998) and LDSS- $3^{28}$ instruments, respectively. The $3.6 \mathrm{~m}$ New Technology Telescope (NTT) located at La Silla was used with the EFOSC2 $2^{29}$ instrument, and the Southern Astrophysical Research (SOAR) $4.1 \mathrm{~m}$ telescope located at Cerro Pachón was used with the Goodman spectrograph (Clemens et al. 2004). Additional late-time spectra were obtained with the Very Large Telescope (VLT) with the FOcal Reducer and low-dispersion Spectrograph (FORS2 ${ }^{30}$ ).

Reduction of all spectra followed standard procedures using the IRAF/PyRAF software. SALT data were first processed by the PySALT ${ }^{31}$ pipeline (see Crawford et al. 2010). Wavelength calibrations were made with arc lamps and verified with the night-sky lines. Relative flux calibrations were made from observations of spectrophotometric standard stars from Oke (1990) and Hamuy et al. (1992, 1994). Gaps between CCD chips have been interpolated in instances when dithering between exposures was not possible, and cosmetic defects have been cleaned manually.

A recession velocity of $2780 \mathrm{~km} \mathrm{~s}^{-1}$ determined from coincident nebular $\mathrm{H} \alpha$ emission has been removed from the presented spectra. This velocity is in agreement with a previous measurement of $2792 \pm 4 \mathrm{~km} \mathrm{~s}^{-1}$ reported by Koribalski et al. (2004) using radio H I lines from the host NGC 6925.

\footnotetext{
28 http://www.lco.cl/telescopes-information/magellan/instruments-1/ldss-3-1/

29 http://www.eso.org/sci/facilities/lasilla/instruments/efosc/

$30 \mathrm{http} / / / \mathrm{www} . e s o . o r g / \mathrm{sci} /$ facilities/paranal/instruments/fors/

31 http://pysalt.salt.ac.za/
}

\subsection{Radio Observations with the VLA}

We also obtained radio observations of SN 2011ei beginning shortly after explosion. On 2011 August 3.2, we observed SN 2011ei with the Karl G. Jansky VLA (Perley et al. 2011) at frequencies of $v=5$ and $25 \mathrm{GHz}$. Following a strong detection of radio emission at $5 \mathrm{GHz}$ (Chomiuk \& Soderberg 2011), we initiated a VLA monitoring campaign at frequencies spanning 2-9 GHz to obtain observations between August 2011 and March 2012 (PI: Soderberg; 10C-168, 11B-212).

All VLA observations were obtained with the widest bandwidth available at that time during the commissioning phase. Prior to 2011 October 1 this amounted to two $128 \mathrm{MHz}$ wide sub-bands (total bandwidth of $256 \mathrm{MHz}$ ), while we later utilized the total bandwidth of $2 \mathrm{GHz}$ at $S$ and $C$ bands; in the latter case the central frequencies were tuned to 5.0 and $6.8 \mathrm{GHz}$. Given that fully upgraded $X$-band receivers were not yet available, our observations at this band spanned 8.0-8.8 GHz. We used J2101-2933 as a phase-calibrator source and 3C48 for flux calibration. Initial observations were obtained in the extended A-configuration while later observations were obtained in the compact C- and D-configurations.

Data were reduced using the standard packages of the Astronomical Image Processing System. In each observation, we fit a Gaussian to the source and extracted the integrated flux density. For observations in the compact D-array we limited the uv-range ( $\gtrsim 5 \mathrm{k} \lambda$ ) to minimize contamination from diffuse host galaxy emission. Our final flux density measurements for SN 2011ei are reported in Table 4. 
Table 4

VLA Observations of SN 2011ei

\begin{tabular}{|c|c|c|c|}
\hline $\begin{array}{l}\text { Date } \\
\text { (UT) }\end{array}$ & $\begin{array}{c}v \\
(\mathrm{GHz})\end{array}$ & $\begin{array}{c}F_{v} \\
(\mu \mathrm{Jy})\end{array}$ & $\begin{array}{c}\text { Array } \\
\text { Config. }\end{array}$ \\
\hline 2011 Aug 3.2 & 5.0 & $93 \pm 25$ & A \\
\hline$\ldots$ & 25 & $130 \pm 140$ & $\ldots$ \\
\hline 2011 Aug 5.2 & 5.0 & $143 \pm 30$ & $\mathrm{~A}$ \\
\hline 2011 Aug 7.2 & 3.1 & $37 \pm 45$ & $\mathrm{~A}$ \\
\hline$\ldots$ & 5.0 & $226 \pm 38$ & $\ldots$ \\
\hline$\ldots$ & 8.5 & $589 \pm 38$ & $\ldots$ \\
\hline 2011 Aug 12.2 & 3.1 & $143 \pm 45$ & A \\
\hline$\ldots$ & 5.0 & $289 \pm 33$ & $\ldots$ \\
\hline$\ldots$ & 8.5 & $589 \pm 38$ & $\ldots$ \\
\hline 2011 Aug 17.3 & 3.1 & $289 \pm 68$ & A \\
\hline$\ldots$ & 5.0 & $564 \pm 33$ & $\ldots$ \\
\hline$\ldots$ & 8.5 & $743 \pm 38$ & $\ldots$ \\
\hline 2011 Aug 25.2 & 3.1 & $353 \pm 52$ & A \\
\hline$\ldots$ & 5.0 & $511 \pm 74$ & $\ldots$ \\
\hline 2011 Sep 8.1 & 3.1 & $497 \pm 65$ & A \\
\hline$\ldots$ & 5.0 & $279 \pm 50$ & $\ldots$ \\
\hline$\ldots$ & 8.5 & $182 \pm 47$ & $\ldots$ \\
\hline 2011 Oct 11.0 & 5.0 & $411 \pm 22$ & $\mathrm{D}$ \\
\hline$\ldots$ & 6.8 & $332 \pm 19$ & $\ldots$ \\
\hline$\ldots$ & 8.4 & $290 \pm 26$ & $\ldots$ \\
\hline 2011 Dec 3.9 & 5.0 & $256 \pm 30$ & $\mathrm{D}$ \\
\hline$\ldots$ & 6.8 & $168 \pm 19$ & $\ldots$ \\
\hline$\ldots$ & 8.4 & $219 \pm 19$ & $\ldots$ \\
\hline 2012 Jan 29.8 & 5.0 & $272 \pm 26$ & $\mathrm{C}$ \\
\hline$\ldots$ & 6.8 & $197 \pm 18$ & $\ldots$ \\
\hline$\ldots$ & 8.4 & $124 \pm 20$ & $\ldots$ \\
\hline 2012 Mar 10.6 & 2.5 & $268 \pm 41$ & $\mathrm{C}$ \\
\hline$\ldots$ & 3.5 & $330 \pm 30$ & $\ldots$ \\
\hline$\ldots$ & 5.0 & $199 \pm 20$ & $\ldots$ \\
\hline$\ldots$ & 6.8 & $116 \pm 15$ & $\ldots$ \\
\hline$\ldots$ & 8.4 & $130 \pm 16$ & $\ldots$ \\
\hline
\end{tabular}

Table 5

Epoch of Maximum Light and Peak Magnitude for the Light Curves of SN 2011ei

\begin{tabular}{lccc}
\hline \hline Filter & $\begin{array}{c}\text { Peak Time } \\
(\mathrm{UT})\end{array}$ & $\begin{array}{c}\text { Peak Time } \\
(\mathrm{JD}-2400000)\end{array}$ & $\begin{array}{c}\text { Peak Obs. } \\
\left(\mathrm{m}(\lambda)_{\max }\right)\end{array}$ \\
\hline$u v w 1$ & 2011 Aug 05.0 & $55778.5 \pm 2.0$ & $17.8 \pm 0.10$ \\
$u$ & 2011 Aug 06.0 & $55779.5 \pm 1.5$ & $16.6 \pm 0.10$ \\
$B$ & 2011 Aug 10.0 & $55783.5 \pm 2.0$ & $17.4 \pm 0.05$ \\
$V$ & 2011 Aug 13.0 & $55786.5 \pm 1.5$ & $17.0 \pm 0.05$ \\
$R$ & 2011 Aug 13.5 & $55787.0 \pm 1.5$ & $16.8 \pm 0.07$ \\
$I$ & 2011 Aug 16.0 & $55789.5 \pm 1.5$ & $16.6 \pm 0.07$ \\
\hline
\end{tabular}

\section{PROPERTIES OF THE UV, OPTICAL, AND BOLOMETRIC LIGHT CURVES}

\subsection{UV and Optical Light Curves and Colors}

Figure 3 shows the observed Swift-UVOT and PROMPT light curves of SN 2011ei. The properties of these curves are presented in Table 5. The time and observed magnitude at maximum light of each filter light curve were estimated with low-order polynomials. The average time to rise to peak brightness from the time of explosion $\left(t_{\exp }\right)$ for $\mathrm{SNe}$ IIb and Ib in the $V$ band is $\Delta t_{\exp } \lesssim 20$ days (Richardson et al. 2006; Drout et al. 2011). Thus, our discovery observation is likely to have been obtained within $\sim 1$ day of outburst and we adopt an explosion date of July 25.0 hereafter.

In Figure 4, the absolute $V$-band light curve of SN 2011ei is compared with those of well-observed SNe IIb and Ib. With a

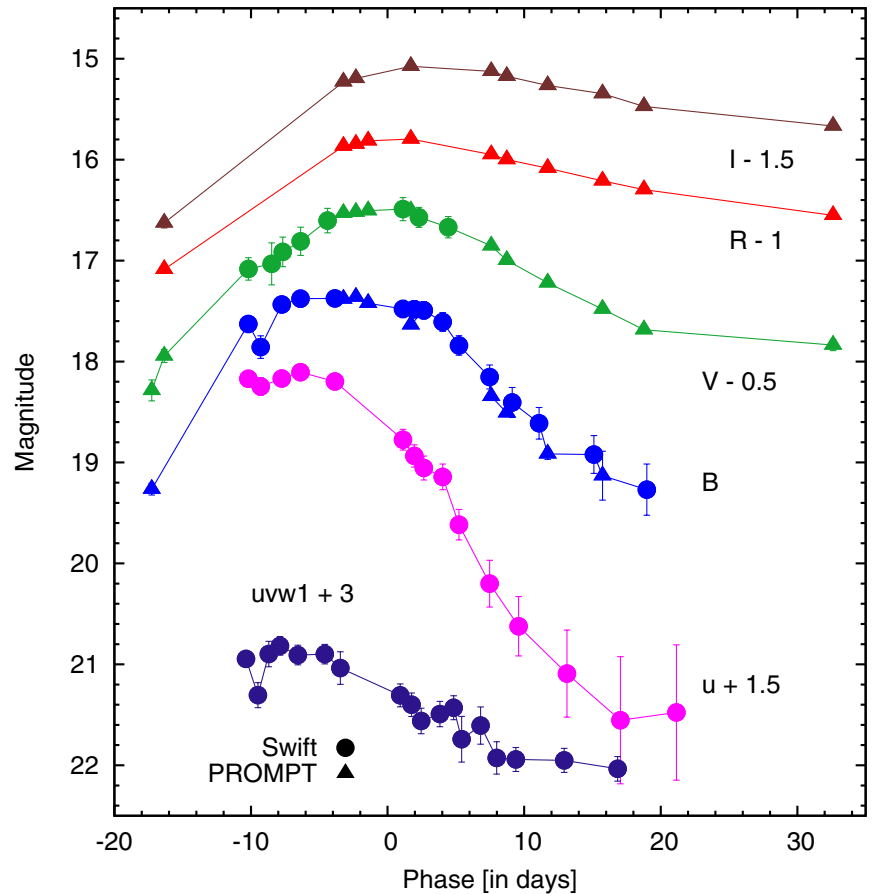

Figure 3. Swift-UVOT and PROMPT light curves of SN 2011ei. Light curves have been shifted by the indicated amounts for clarity.

(A color version of this figure is available in the online journal.)

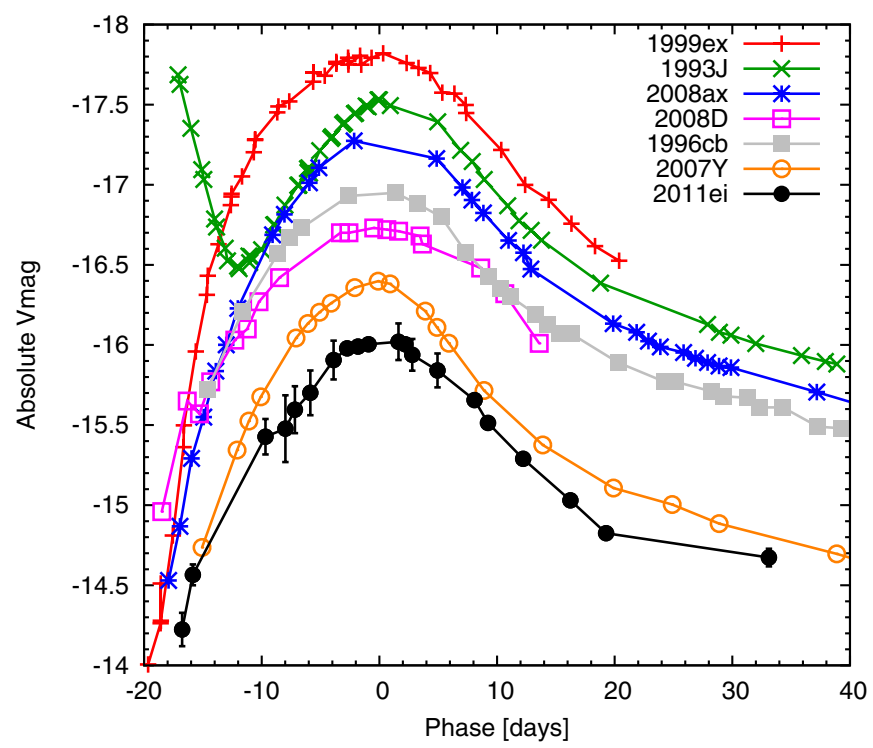

Figure 4. $V$-band absolute light curve of SN 2011ei compared to light curves of Type IIb and Ib objects: SN 1999ex (Type Ib; Stritzinger et al. 2002), SN 1993J (Type IIb; Lewis et al. 1994), SN 2008ax (Type IIb; Pastorello et al. 2008), SN 1996cb (Type IIb; Qiu et al. 1999), and SN 2007Y (Type Ib; Stritzinger et al. 2009).

(A color version of this figure is available in the online journal.)

peak absolute magnitude of $M_{V} \approx-16$ mag, SN 2011ei was approximately 2 mag below the mean peak absolute magnitudes of the entire class of stripped-envelope events (Richardson et al. 2006; Drout et al. 2011). Other low-luminosity events include the Type Ib SN 2007Y $\left(M_{V}=-16.5\right.$; Stritzinger et al. 2009) and SN 2008D $\left(M_{V}=-16.7\right.$; Soderberg et al. 2008).

In Figure 5, we plot the observed $(B-V)$ color curve of SN 2011ei with respect to $V$-band maximum along with those of other $\mathrm{SNe} \mathrm{IIb}$ and Ibc. Also shown are the same color 

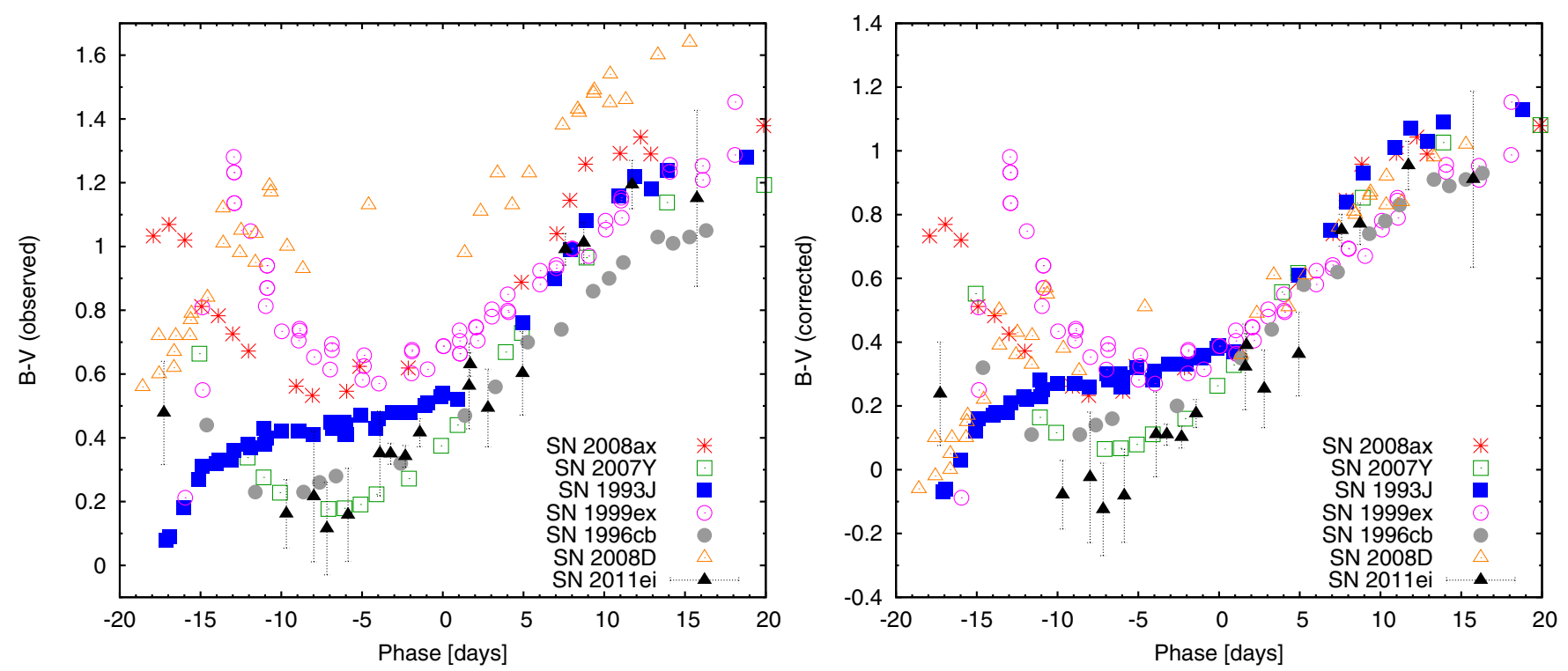

Figure 5. $B-V$ color curves of SN 2011ei compared to those of other stripped-envelope SNe: SN 2008ax (Type IIb; Pastorello et al. 2008), SN 2007Y (Type Ib; Stritzinger et al. 2009), SN 1993J (Type IIb; Lewis et al. 1994), SN 1999ex (Type Ib; Stritzinger et al. 2002), SN 1996cb (Type IIb; Qiu et al. 1999), and SN 2008D (Type Ib; Mazzali et al. 2008). On the left are observed colors, and on the right are colors corrected for extinction.

(A color version of this figure is available in the online journal.)

curves corrected for extinction. Like SN 1999ex and SN 2008ax, the $(B-V)$ colors of SN 2011ei first become increasingly blue in the days immediately following outburst. Then, around 5-10 days before maximum, the SN becomes redder with time and the color indices increase monotonically. The majority of $(B-V)$ color indices of SN 2011ei are somewhat bluer than those of other $\mathrm{SNe}$ after correction. This blue excess suggests that the adopted $E(B-V)=0.24$ mag is likely an upper limit.

\subsection{Bolometric Light Curve}

The extinction-corrected Swift-UVOT and PROMPT light curves were combined to obtain a quasi-bolometric light curve $\left(L_{\mathrm{bol}}^{\text {quasi }}\right)$ of SN 2011ei. Low-order polynomials have been used to interpolate the light curves. The total $\mathrm{UV}+B V R I$ flux was determined by summing the integrated fluxes of the different filters and uncertainties have been propagated following standard practice.

The quasi-bolometric $\mathrm{UV}+B V R I$ light curve was then transformed into a bolometric light curve assuming $L_{\text {bol }}^{\text {quasi }} \approx 0.8 L_{\text {bol }}$ and that $0.2 L_{\text {bol }}$ is emitted as unobserved near-infrared emission. This estimate is in line with bolometric reconstructions of $\mathrm{SNe} \mathrm{IIb}$ and Ib with extensive observations such as SN 2007Y (Stritzinger et al. 2009), SN 2008ax (Pastorello et al. 2008; Taubenberger et al. 2011), and SN 2008D (Mazzali et al. 2008; Soderberg et al. 2008).

The final bolometric light curve of SN 2011ei is shown in Figure 6. Also shown in the figure are the bolometric light curves of SN 1999ex, 1993J, 2007Y, and 2008D. The bolometric light curve peaks $\approx 14.4$ days after outburst with $L_{\text {bol }} \sim$ $7 \times 10^{41} \mathrm{erg} \mathrm{s}^{-1}$, making SN 2011ei one of the least luminous $\mathrm{SNe} \mathrm{IIb} / \mathrm{Ib}$ observed to date. Among the closest in luminosity is SN 2007 Y which peaked at $L_{\text {bol }} \sim 1.3 \times 10^{42} \mathrm{erg} \mathrm{s}^{-1}$.

\subsection{Explosion Parameters}

We modeled the bolometric light curve to infer the ejecta mass $\left(M_{\mathrm{ej}}\right)$, the nickel mass $\left(M_{\mathrm{Ni}}\right)$, and the kinetic energy of the ejecta $\left(E_{k}\right)$. Following the procedures of Valenti et al. (2008),

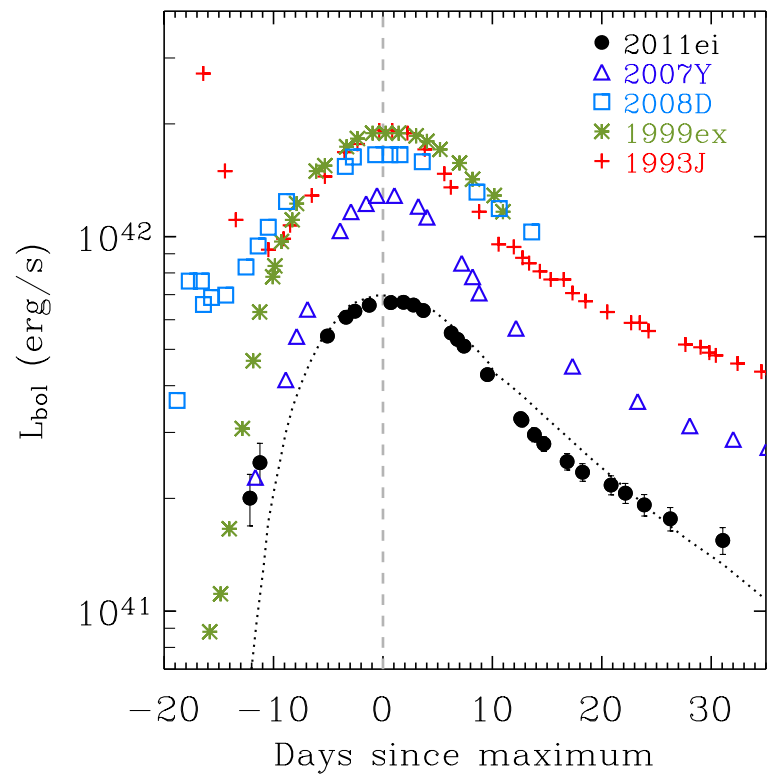

Figure 6. Reconstructed bolometric light curve of SN 2011ei compared to those of SN 2007Y (Type Ib; Stritzinger et al. 2009), SN 2008D (Type Ib; Mazzali et al. 2008), SN 1999ex (Type Ic; Stritzinger et al. 2002), and SN 1993J (Type IIb; Richmond et al. 1994). Plotted over the points for SN 2011ei is the final best-fit curve used to determine explosion parameters. Phase is with respect to the peak of SN 2011ei's bolometric light curve, which occurs on 2011 August 08.4 (JD 2456147.9).

(A color version of this figure is available in the online journal.)

we assumed the early-time $\left(\Delta t_{\exp }<40\right.$ days) light curve to correspond with the photospheric regime and adopted a constant optical opacity $k_{\mathrm{opt}}=0.07 \mathrm{~cm}^{2} \mathrm{~g}^{-1}$, which is valid so long as electron scattering is the dominant opacity source and the ejecta are triply ionized (see, e.g., Chugai 2000). At late times $\left(\Delta t_{\text {exp }}>40\right.$ days) the optical depth of the ejecta decreases and the observed luminosity is powered by $\gamma$-rays arising from the ${ }^{56} \mathrm{Co}$ decay, $\gamma$-rays from electron-positron annihilation, and the 
kinetic energy of the positrons (Sutherland \& Wheeler 1984; Cappellaro et al. 1997).

From the above modeling the following best-fitting parameters were derived: $M_{\mathrm{ej}} \sim 1.6 M_{\odot}, E_{k} \sim 2.5 \times 10^{51} \mathrm{erg}$, and $M_{\mathrm{Ni}}=0.030 \pm 0.010 M_{\odot}$. Our model underpredicts the observed luminosity at early times $\left(\Delta t_{\exp }<5\right.$ days). This suggests the presence of an additional component of emission. We explore the possibility of cooling envelope emission following shock break-out in Section 3.4.

As an independent check we note that our best-fitting explosion parameters imply a photospheric velocity, $v_{\mathrm{ph}}$, at peak luminosity of

$$
v_{\mathrm{ph}} \approx\left(\frac{6 E_{k}}{5 M_{e j}}\right)^{0.5} \sim 10,000 \mathrm{~km} \mathrm{~s}^{-1},
$$

which is in agreement with the expansion velocities observed in the optical spectra (see Section 4.1). A separate check of the Ni mass comes from a relationship between $M_{\mathrm{Ni}}$ versus peak absolute $R$-band magnitude $\left(M_{R}\right)$ presented in Drout et al. (2011). Given SN 2011ei's extinction-corrected peak of $M_{R} \approx-16.1 \mathrm{mag}$, this relationship yields an Ni mass of $M_{\mathrm{Ni}}<0.04 M_{\odot}$, which is consistent with our result.

\subsection{Limits on the Radius of the Progenitor Star}

The fortuitous discovery of SN 2011ei not long after outburst enabled us to trace the temporal evolution of the early optical emission (see Figure 3). As discussed by Ensman \& Burrows (1992), this is the time of cooling envelope emission after shock breakout, and provides a good indicator of the initial stellar extent.

A significant difference between SN 2011ei and SN 1993J clearly illustrated in Figure 4 is that SN 2011ei does not show the prominent early-time peak exhibited by the light curve of SN 1993J. This peak is attributed to the initial shock heating and subsequent cooling of a low-mass envelope of SN 1993J's progenitor star of radius $\sim 4 \times 10^{13} \mathrm{~cm}$ (Bartunov et al. 1994; Shigeyama et al. 1994; Woosley et al. 1994). The absence of a comparable early luminous phase in SN 2011ei suggests its progenitor star had a smaller envelope than that of SN 1993J.

The first $R$-band detection of SN 2011ei was made shortly after explosion with luminosity, $L_{R} \approx 2.4 \times 10^{40} \mathrm{erg} \mathrm{s}^{-1}$. This initial observation was used to constrain the radius of the progenitor star by modeling the cooling envelope emission adopting the formalism of Chevalier \& Fransson (2008). In this model the emission is roughly approximated as a blackbody spectrum with a radius and temperature determined by the ejecta parameters, $M_{\mathrm{ej}}$ and $E_{k}$ (see Chevalier \& Fransson 2008 and Nakar \& Sari 2010 for a detailed discussion). In turn, these parameters enable an estimate of the progenitor radius, $R_{*}$. In this scenario, the temperature of the photosphere is

$$
T_{\mathrm{ph}} \approx 7800 E_{k, 51}^{0.03} M_{\mathrm{ej}, \odot}^{-0.04} R_{*, 11}^{0.25} \Delta t_{\mathrm{exp}}^{-0.48} \mathrm{~K},
$$

and the photospheric radius is

$$
R_{\mathrm{ph}} \approx 3 \times 10^{14} E_{k, 51}^{0.39} M_{\mathrm{ej}, \odot}^{-0.28} \Delta t_{\mathrm{exp}}^{0.78} \mathrm{~cm} .
$$

Here, $E_{k, 51}$ is $E_{k}$ in units of $10^{51} \mathrm{erg}, M_{\mathrm{ej}, \odot}$ is in units of $M_{\odot}$, $R_{*, 11}$ is in units of $10^{11} \mathrm{~cm}$, and $\Delta t_{\exp }$ is time in days since explosion.

For explosion parameters determined in Section 3.3 and the adopted explosion date 2011 July 25.0, the extinction-corrected discovery magnitude constrains $R_{*}<10^{11} \mathrm{~cm}$. This estimate is consistent with upper limits on the progenitor radius derived from a similar analysis of the first $B$ - and $V$-band detections $\sim 2.5$ days after explosion (see Table 1). We note that the temperature is sufficiently low that recombination is important and the model of Chevalier \& Fransson (2008) is inaccurate. Furthermore, the model predicts a temperature that decreases with time, whereas our earliest photometric and spectroscopic measurements suggest an increasing temperature. However, our estimate of the upper limit to progenitor radius should still be valid to within a factor of 2-3. Thus, the early optical emission points to a compact progenitor, similar to those of SNe Ibc and compact IIb of radius $R_{*} \lesssim 10^{11} \mathrm{~cm}$ (Chevalier \& Soderberg 2010).

\section{OPTICAL SPECTROSCOPY}

We now describe the optical spectra of SN 2011ei and compare their properties to those of other stripped-envelope events. We also use these spectra to probe SN 2011ei's ejecta mass distribution by modeling the SN's late-time emissions. The times of the spectroscopic observations, $t$, are provided relative to the time of $V$-band maximum. Line identifications and estimates of expansion velocities were made with the SN spectrum synthesis code SYNOW. Manual and automated procedures employing the recently updated versions of the software, SYN++ and SYNAPPS, were used. ${ }^{32}$ The basic assumptions of SYNOW include spherical symmetry, velocity proportional to radius, a sharp photosphere, line formation by resonant scattering treated in the Sobolev approximation, local thermodynamic equilibrium (LTE) for the level populations, no continuum absorption, pure resonance scattering, and only thermal excitations. Fits are constrained by how we are able to best match absorption minimum profiles, as well as the relative strengths of all the features (see Branch et al. 2002 for more description of fitting parameters and Thomas et al. 2011 for software details). Representative SYNOW fits are shown in Figure 7.

\subsection{Early Spectral Evolution}

The pre-maximum light optical spectra of SN 2011ei evolved rapidly. The earliest spectrum $(t=-14$ days; Figure 8) shows strong Type II-like hydrogen P-Cygni features. The $\mathrm{H} \alpha$ profile exhibits a "saw-toothed" shape, rising steeply from a minimum at $6220 \AA\left(-16,000 \mathrm{~km} \mathrm{~s}^{-1}\right)$ to a maximum at $6395 \AA$. Redshifted emission extends to approximately $7000 \AA$ $\left(+19,000 \mathrm{~km} \mathrm{~s}^{-1}\right)$. In Figure 8, two SYNOW synthetic fits to the $t=-14$ day spectrum of SN 2011ei are shown: one a complete model, and the other using only hydrogen. The $\mathrm{H} \beta$, $\mathrm{H} \gamma$, and $\mathrm{H} \epsilon$ absorptions are fit reasonably well with a velocity of $14,500 \mathrm{~km} \mathrm{~s}^{-1}$. Additional features in this spectrum are fit with $\mathrm{Ca}$ II, Na I, and Fe II. Attempts to produce satisfactory fits of the $\mathrm{H} \alpha$ profile failed, likely due to the LTE and resonance scattering assumptions of SYNOW.

In the next two spectra on $t=-10$ days and $t=-6$ days, the prominent $\mathrm{H} \alpha$ emission profile subsides and the spectra instead exhibit conspicuous He absorptions usually associated with $\mathrm{SNe}$ Ib. The continuum becomes increasingly blue and the He I $\lambda 5016, \lambda 5876, \lambda 6678$, and $\lambda 7065$ lines appear but are weak and narrow. Blueshifted absorption of the $\mathrm{H} \alpha$ line around $6250 \AA$ develops two minima. The center of the trough sits around the

\footnotetext{
32 Software was retrieved from https://c3.lbl.gov/es/
} 


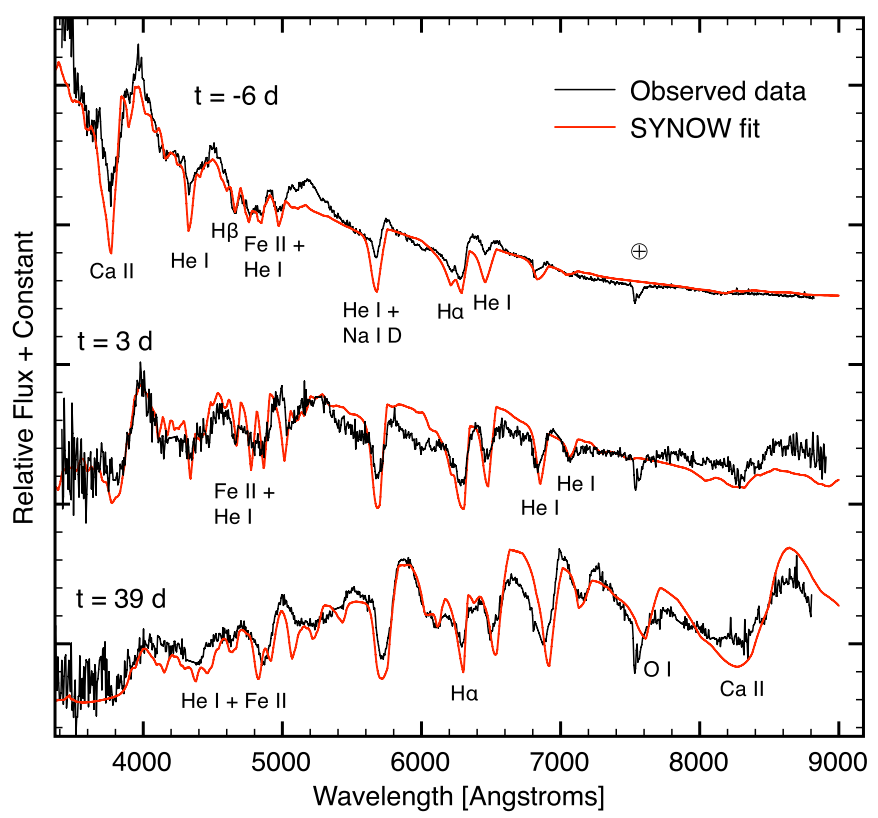

Figure 7. Representative SYNOW fits to optical spectra of SN 2011ei. Phase is with respect to $V$-band maximum.

(A color version of this figure is available in the online journal.)

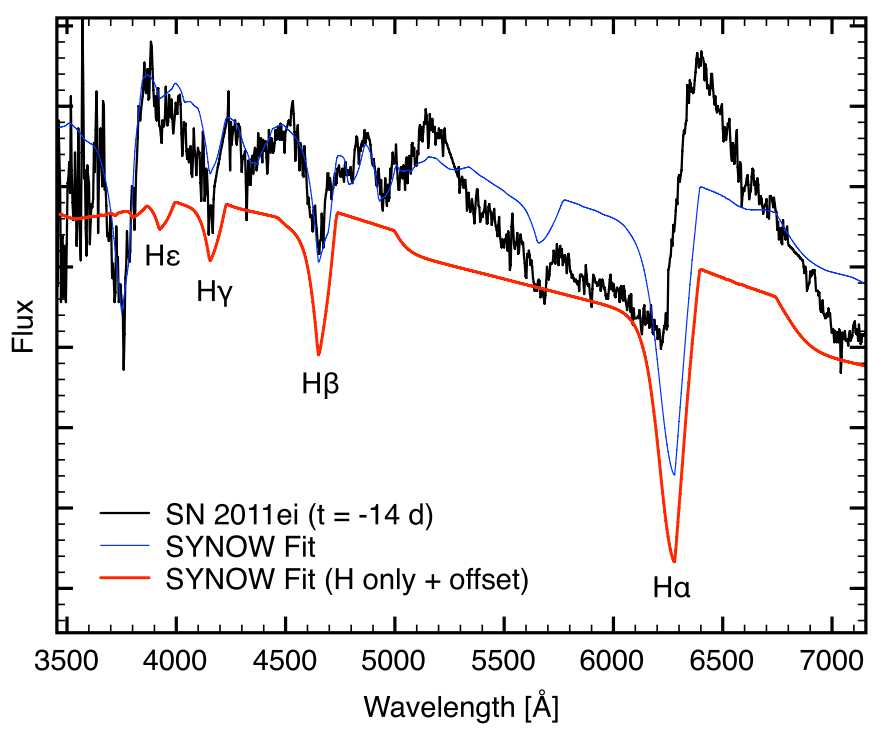

Figure 8. Attempted SYNOW fits of $t=-14$ day spectrum of SN 2011ei. The blue line represents the complete model and the red line represents the model only using hydrogen. Features blueward of $5000 \AA$ are fit reasonably well, but none of the models we tried fit the broad $\mathrm{H} \alpha$ profile.

(A color version of this figure is available in the online journal.)

observed wavelength of $6300 \AA$ so contamination from the [O I] $\lambda 6300$ night-sky line is possible. However, the peak between the two absorptions is fairly strong and too broad to be associated with narrow, unresolved telluric emission.

The $6250 \AA$ feature was modeled using two components of hydrogen at velocities of $\approx 17,000 \mathrm{~km} \mathrm{~s}^{-1}$ and $14,000 \mathrm{~km} \mathrm{~s}^{-1}$. Though two distinct absorptions are observed around $\mathrm{H} \alpha$, only one conspicuous absorption is observed around $\mathrm{H} \beta$. The SYNOW fit confirms that two components of hydrogen with a velocity difference of $\sim 3000 \mathrm{~km} \mathrm{~s}^{-1}$ blend together and manifest as a single feature in all Blamer lines except $\mathrm{H} \alpha$. Attempts to fit with other ions such as $\mathrm{Si}$ II, Ne I, and $\mathrm{C}$ II introduced inappropriate features elsewhere in the synthetic spectra and/or

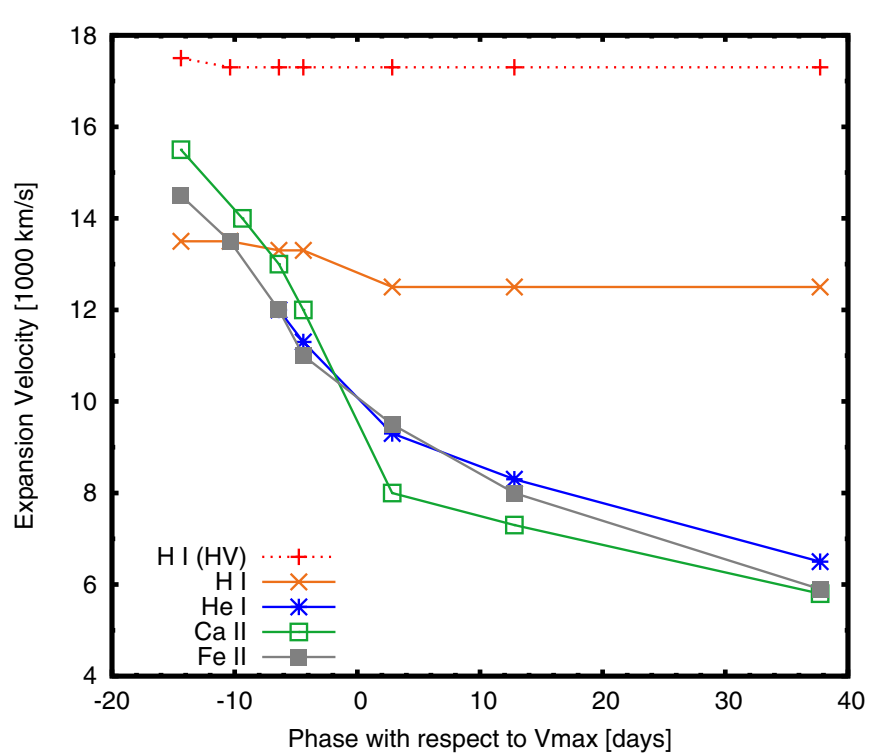

Figure 9. Expansion velocities of prominent ions in the spectra of SN 2011ei determined from SYNOW fitting. Uncertainties in velocity estimates are $\sim 500 \mathrm{~km} \mathrm{~s}^{-1}$. The identification of high-velocity (HV) $\mathrm{H}$ I is poorly constrained since it is observed only in $\mathrm{H} \alpha$.

(A color version of this figure is available in the online journal.)

were at velocities well below the photosphere and were considered unsuccessful. The properties of this absorption as observed in SN 2011ei and a number of stripped-envelope events are explored further in Section 4.5.

From $t=-6$ to $t=+3$ days, the He I lines strengthen. High-velocity calcium absorption is not detected in the early spectra, which is interesting given that it has been reported at these epochs in several SNe IIb and Ib including SN 1999ex, SN 2005bf, SN 2007Y, and SN 2008ax (Stritzinger et al. 2002; Folatelli et al. 2006; Stritzinger et al. 2009), but not in SN 2008D (Modjaz et al. 2009). Emission around $8700 \AA$ Associated with the infrared Ca II triplet is noticeable shortly after maximum light and then gradually strengthens in the following months.

Additional ions other than $\mathrm{HI}, \mathrm{He}$ I, Ca II, Na I, and Fe II were introduced to the synthetic spectra to model the $t=$ 39 day spectrum. The most conspicuous change is $\mathrm{O}$ I absorption around $7600 \AA$. Additional absorptions are fit using a blend of $\mathrm{S}_{\text {II }}$ features around $5200 \AA$, Si II $\lambda 6355$, and Ba II $\lambda 6142$ and $\lambda 6496$; however, their removal does not change any conclusions reported here and they are not considered significant.

The evolution in the expansion velocities for the most prominent ions in SN 2011ei as estimated from the SYNOW fitting is shown in Figure 9. Some noteworthy trends are observed. The high-velocity hydrogen shows almost no change over all of the sampled epochs, and the second, lower-velocity hydrogen component shows a slight drop before leveling out after maximum light. The remaining ions $\mathrm{He}$ I, Ca II, and Fe II show velocities that drop most steeply before maximum light, after which the rate of velocity change decreases. The expansion velocities of the He I and Fe II ions closely follow each other.

\subsection{Comparison to SNe IIb and Ib}

SN 2011ei shares spectral properties with many SNe IIb and Ib. Selected examples are plotted in Figure 10. The earliest spectrum $(t=-14$ days) shows close similarity to that of SN 2007Y (Stritzinger et al. 2009). Both SNe exhibit a rarely observed, extended, high-velocity $\mathrm{H} \alpha$ profile that dominates 


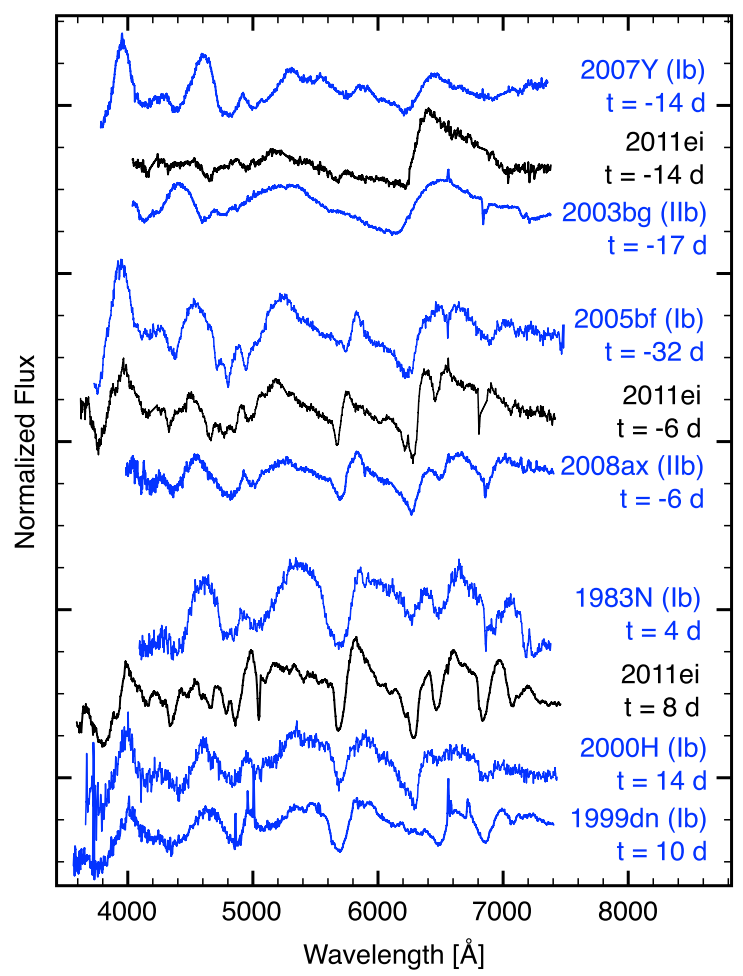

Figure 10. Early spectra of SN 2011ei compared to those of other SNe IIb and Ib. Spectra have been normalized according to the procedure outlined in Jeffery et al. (2007) to aid in visual comparison.

(A color version of this figure is available in the online journal.)

the spectrum. However, SN 2011ei does not have the broad calcium absorption seen in SN $2007 \mathrm{Y}$ around $8400 \AA$, nor does it show the pronounced features between 4000 and $5000 \AA$ associated with Fe II and He I. A likeness between SN 2011ei and SN 2003bg is also seen (Hamuy et al. 2009; Mazzali et al. 2009). In this case, the $\mathrm{H} \alpha$ profiles are comparable, but $\mathrm{SN}$ 2003 bg shows a blueshifted absorption associated with higher velocities $\left(\sim 20,000 \mathrm{~km} \mathrm{~s}^{-1}\right)$ and distributed more gradually. As with SN 2007 Y, the features blueward of $5000 \AA$ are more pronounced in SN 2003bg than in SN 2011ei.

Approximately one week before maximum light when the $\mathrm{H} \alpha$ emission is no longer conspicuous in SN 2011ei, its spectra resemble those of $\mathrm{SNe}$ IIb from compact progenitors and $\mathrm{SNe} \mathrm{Ib}$ exhibiting absorption around $\approx 6250 \AA$. Shown in Figure 10 (middle section) is the Type Ib SN 2005bf (Folatelli et al. 2006) early in its unusual late-peaking light curve, and the Type IIb SN 2008ax (Pastorello et al. 2008) at $t=-6$ days. By the time of maximum light and in the months that follow, SN 2011ei continues to exhibit spectral properties consistent with those of SNe Ib. Other examples plotted in Figure 10 (bottom section) are SN 1983N (Harkness et al. 1987), and SNe 1999dn and 2000H (retrieved electronically via SUSPECT courtesy of the Asiago SN Group). Of all the SNe IIb and Ib examined, the EWs of the hydrogen and helium features in the spectra of SN 2011ei were among the most narrow.

\subsection{Nebular Phase Spectrum}

Starting with the $t=73$ day spectrum, SN 2011ei begins to enter the nebular phase and emission from forbidden transitions is apparent. Emission from the [O I] $\lambda \lambda 6300,6364,[\mathrm{Ca} I \mathrm{II}]$ $\lambda \lambda 7291,7324$, and $\left.\mathrm{Mg}_{\mathrm{I}}\right] \lambda 4571$ lines originating from the inner ejecta is observed. $\mathrm{H} \alpha$ emission attributable to high-velocity

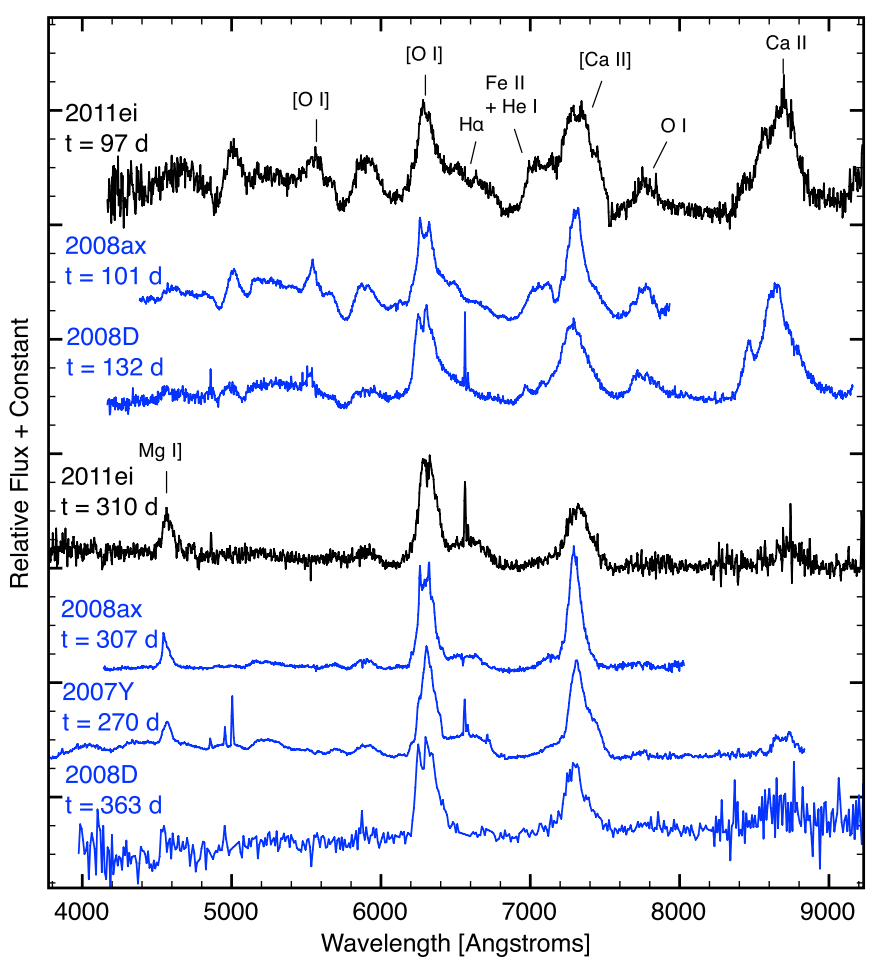

Figure 11. Late-time optical spectra of SN 2011ei compared to spectra of SN 2008ax (Milisavljevic et al. 2010), SN 2007Y (Stritzinger et al. 2009) and SN 2008D $(t=132$ days from Modjaz et al. 2009 and $t=363$ days from Tanaka et al. 2009). O I $\lambda 8446$ may contribute to strong emission observed around the Ca II infrared triplet.

(A color version of this figure is available in the online journal.)

hydrogen gas interacting with dense circumstellar material (CSM; Houck \& Fransson 1996) is also present.

The overall emission properties of SN 2011ei at nebular epochs are quite similar to those of other $\mathrm{SNe} \mathrm{IIb}$ and Ib. This is clear in Figure 11, where the $t=97$ day and $t=310$ day spectra of SN 2011ei are compared to spectra of SN 2007Y, SN 2008D, and SN 2008ax. The ratio of [CaII]/[OI] emission, which has been predicted to be a potential diagnostic of progenitor core mass (Fransson \& Chevalier 1989), is relatively small in SN 2011ei. Unlike SN 2008D and SN 2008ax, SN 2011ei does not show conspicuous multiple peaks in its [O I] $\lambda \lambda 6300,6364$ emission (see, e.g., Mazzali et al. 2005; Modjaz et al. 2008, 2009; Maeda et al. 2008; Taubenberger et al. 2009; Milisavljevic et al. 2010). Thus, the degree of ejecta asymmetry may be less in SN 2011ei compared to those events.

In Figure 12, emission line profiles of the $t=97$ day and $t=310$ day spectra of SN 2011ei are enlarged and plotted in velocity space. The fairly broad [Ca II] $\lambda \lambda 7291,7324$ emission is centered roughly around zero velocity (assumed to lie at $7306 \AA$ ). A blueward ledge that is probably a combination of He I $\lambda 7065$ and $[\mathrm{Fe} \mathrm{II}] \lambda 7155$ diminishes between the two epochs. Close inspection reveals a non-uniform central peak in the [O I] $\lambda \lambda 6300,6364$ profile in the form of a narrow gap centered around $6305 \AA$. The reality of this feature is certain as it appears in both epochs, and interestingly, emission redward of the gap appears to grow stronger with time. The observed substructure is suggestive of clumpy ejecta (see, e.g., Spyromilio 1994), and the increase in redshifted emission may be associated with declining optical opacity (Taubenberger et al. 2009). Alternatively, the profile may be influenced by the high-velocity $\mathrm{H} \alpha$ absorption observed at early epochs (Maurer et al. 2010). 


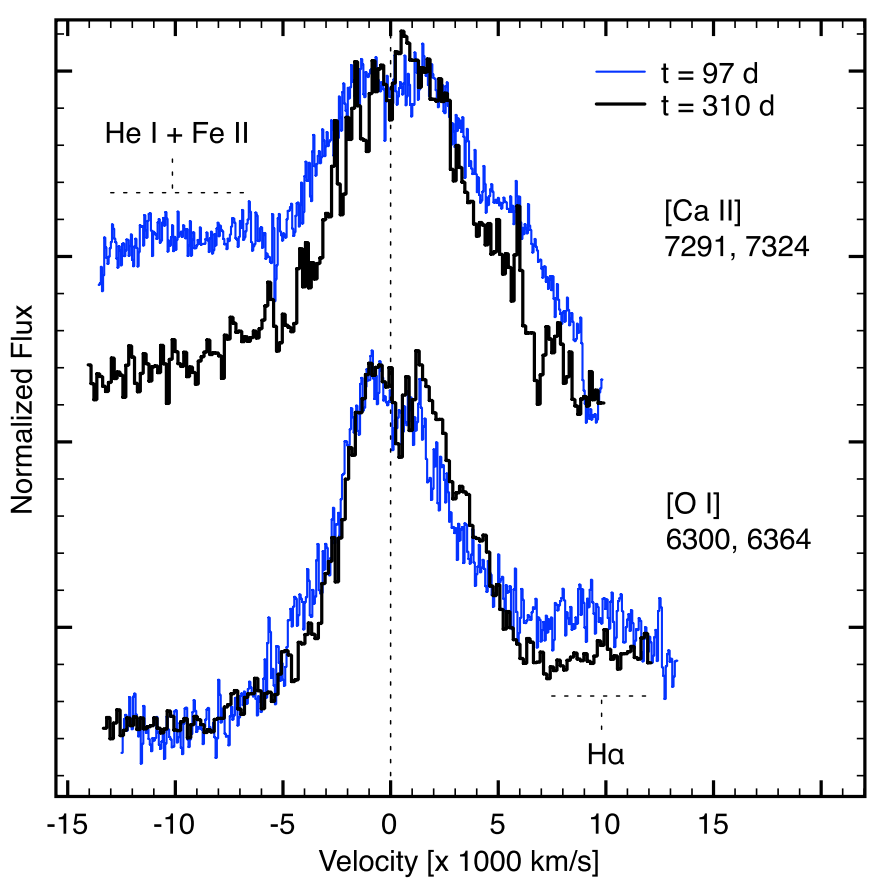

Figure 12. Late-time emission line profiles of SN 2011ei from the $t=97$ day and $t=310$ day spectra. Instances where line profiles are contaminated by emissions from other lines are indicated.

(A color version of this figure is available in the online journal.)

\subsection{Model of Nebular Spectrum}

We modeled the nebular spectrum of SN 2011ei using our SN nebular spectrum code, assuming that the late-time emission is tied to the deposition of gamma-rays and positrons from ${ }^{56} \mathrm{Co}$ decay. Given an ejected mass, a characteristic boundary velocity (which corresponds to the half-width at half-maximum of the emission lines), and a composition, the code computes gammaray deposition, follows the diffusions of the gamma-rays and the positrons with a Monte Carlo scheme and computes the heating of the gas. The state of the gas is then computed in NLTE, balancing heating and cooling via line emission. The code has been used for a number of SNe Ibc (e.g., Mazzali et al. 2001) and is the latest version described in some detail in Mazzali et al. (2011).

For SN 2011ei, we modeled the 2012 June 18 spectrum assuming a rest-frame epoch of $\approx 325$ days (Figure 13 ). We used a line width of $4500 \mathrm{~km} \mathrm{~s}^{-1}$, a distance modulus $\mu=32.27$, and a reddening $E(B-V)=0.24$ (see Section 2.1). We also assumed that the gas has a spherical distribution, which is reasonable given that the line profiles do not seem to indicate the need for large asymmetries (see Section 4.3).

We found that the spectrum (excluding the $\mathrm{H} \alpha$ line, which is mostly the result of recombination) can be powered by an ${ }^{56} \mathrm{Ni}$ mass of $0.047 \pm 0.005 M_{\odot}$. This is determined mainly by fitting the Fe forbidden emission lines, and simultaneously the other lines. The overall ejecta mass is quite small, $\sim 0.3 M_{\odot}$, most of which is oxygen $\left(0.21 M_{\odot}\right)$. Carbon, magnesium, silicon, and sulphur are also present. The $\mathrm{Ca}$ abundance is small $\left(0.002 M_{\odot}\right)$ but this is sufficient to form strong emission lines.

The ejected mass we derived with the nebular model is smaller than our other mass estimate of $1.6 M_{\odot}$ derived from the bolometric light curve (see Section 3.3). Hydrogen can be responsible for another $0.1 M_{\odot}$ or so, as is typical of $\mathrm{SNe} \mathrm{IIb}$ (James \& Baron 2010; Hachinger et al. 2012), but even so,

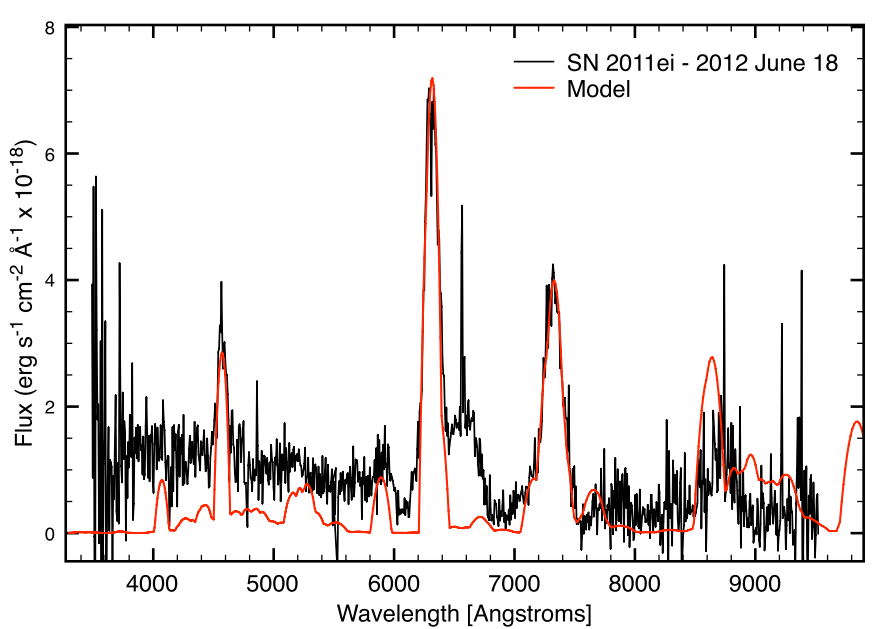

Figure 13. Late-time spectrum of SN 2011ei (black) and our nebular emission model (red).

(A color version of this figure is available in the online journal.)

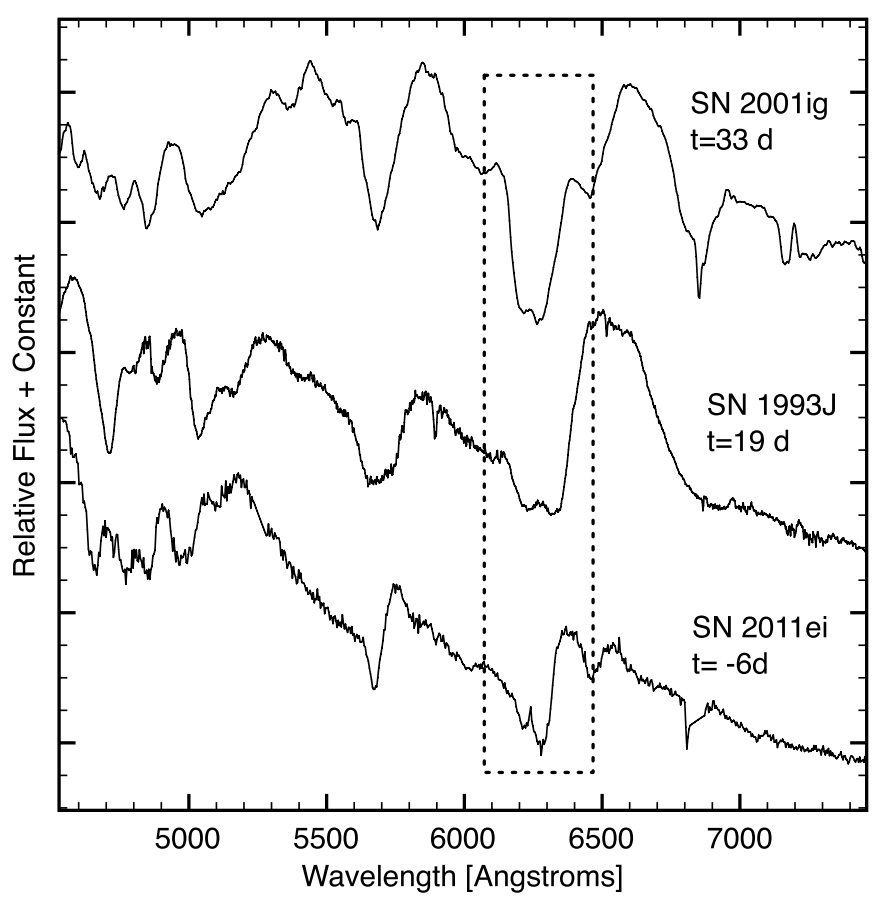

Figure 14. Evidence of two-component absorption centered near $\approx 6250 \AA$ for SN 2011ei, SN 1993J (Matheson et al. 2000), and SN 2001ig (Silverman et al. 2009). Phase is with respect to $V$-band maximum. The dashed box highlights the region around $\mathrm{H} \alpha$ where the absorption is observed.

the total is significantly below $1.6 M_{\odot}$. Possibly some of the outer O-rich ejecta are too thin to cause the efficient deposition of gamma-rays, thus the nebular emission model may have underestimated the mass in this way. Our estimate for $M_{\mathrm{Ni}}$ from nebular modeling, however, is in statistical agreement with the estimate from the peak of the light curve, and we conclude that the Ni mass likely lies within the range of the two estimates; i.e., $0.03 M_{\odot} \lesssim M_{\mathrm{Ni}} \lesssim 0.05 M_{\odot}$.

\subsection{Multi-component Absorption Due to Hydrogen}

The absorption with two minima observed around $6200-6300 \AA$ is not unique to SN 2011ei. In Figure 14, we show spectra of SN 2011ei, SN 1993J, and SN 2001ig that illustrate how these events all share broad absorption suggestive 


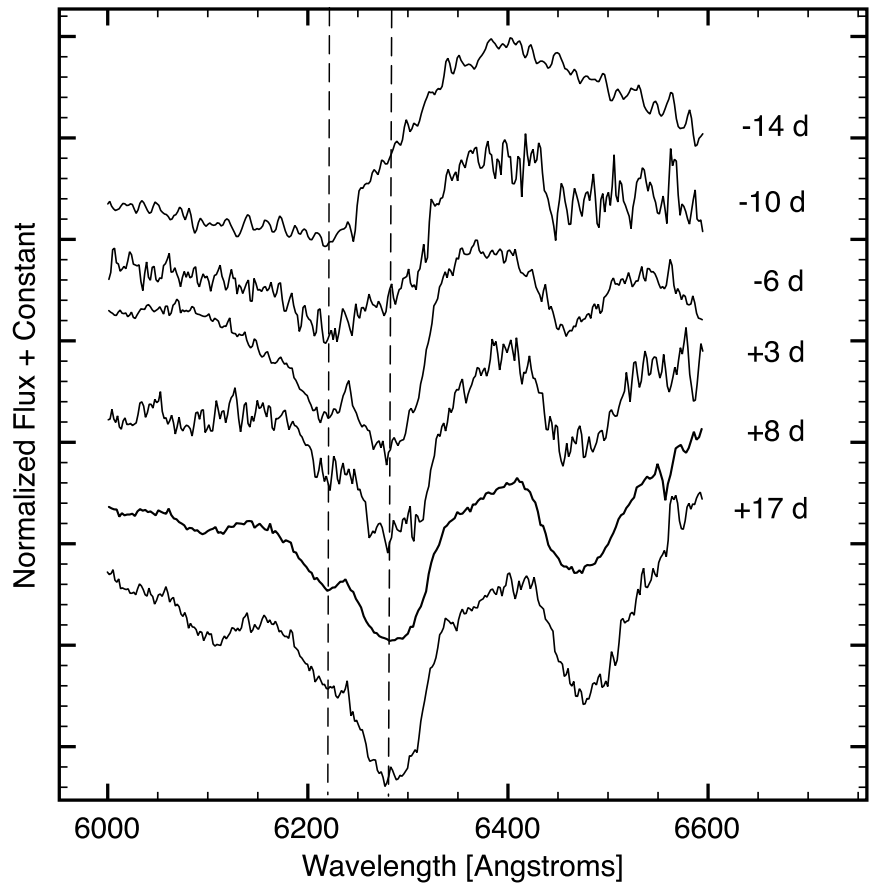

Figure 15. Time series spectra of SN 2011ei in the region of $\mathrm{H} \alpha$ showing evidence for two-component absorption evolution. Phase is with respect to $V$-band maximum. The dashed lines mark the individual components.

of two components. The epoch at which this phenomenon is visible ranges considerably from $t=33$ days in SN 2001ig, to $t=19$ days in SN 1993J, and to $t=-6$ days in SN 2011ei. In the case of SN 2011ei, there is clear evolution in the relative strength of the two components. The blueward absorption is observed first, then the second, redward absorption appears and increases in strength (see Figure 15).

The origin of the two-component feature is not clear. In SN 1993J, its nature has been investigated thoroughly using SYNOW spectral fitting and NLTE modeling (Baron et al. 1994, 1995; Zhang et al. 1995a, 1995b; Zhang \& Wang 1996). Suspected origins include ejecta asymmetry, mixture with Fe II or Si II lines, and a complicated two-component density structure in H-rich material. James \& Baron (2010) found that they were unable to model a similar two-component feature in SN $2000 \mathrm{H}$ and attributed it to interaction with CSM. They cited a quickly fading $\mathrm{Na}$ I $\mathrm{D}$ feature observed around maximum light as evidence for environmental interaction. More recently, Hachinger et al. (2012) attributed the feature in SNe IIb and Ib to a blend of high-velocity $\mathrm{H} \alpha$ and $\mathrm{Si}$ II.

Multi-component absorption may be a common phenomenon across SN types and emission lines. For example, evidence for two components of high-velocity hydrogen absorption has been seen in early spectra of the Type Ib SN 1999dn (Benetti et al. 2011), as well as the Type IIb SN 2011dh during the first month following outburst (H. Marion 2012, private communication). Multi-component absorption has also be recognized in the complex evolution of broad Si II $\lambda 6355$ and $\mathrm{Ca}$ II H\&K absorption features in Type Ia SNe (Foley et al. 2011, 2012).

\subsection{Explosion Site Metallicity}

Possible metallicity differences between SN subtypes and their relation to progenitor systems are currently an active area of investigation (e.g., Prieto et al. 2008; Arcavi et al. 2010; Anderson et al. 2010; Modjaz et al. 2011; Kelly \& Kirshner

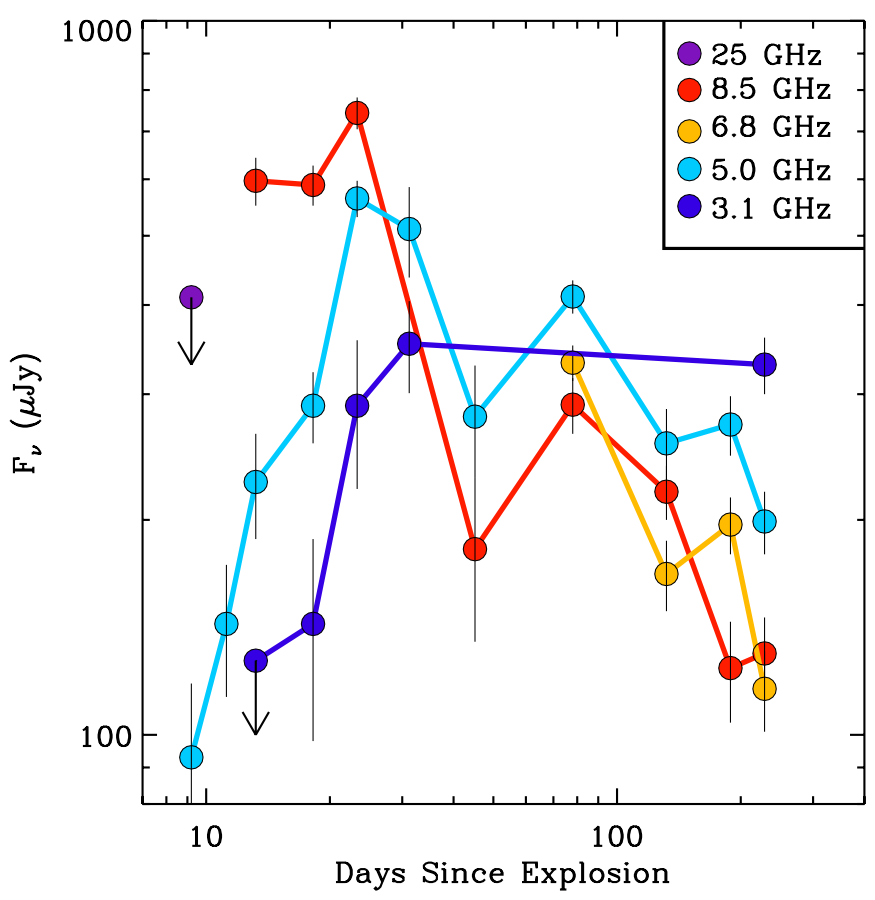

Figure 16. VLA radio light curves of SN 2011ei are shown at frequencies spanning 3.1-25 GHz. At the best-sampled frequency, $5 \mathrm{GHz}$, we find evidence for a secondary maximum at $\Delta t_{\exp } \approx 80$ days that is also seen in the $8.4 \mathrm{GHz}$ light curve.

(A color version of this figure is available in the online journal.)

2012; Sanders et al. 2012). We were therefore motivated to estimate the metallicity of the host environment of SN 2011ei.

In our 2012 June 18 spectrum of SN 2011ei, we detect narrow nebular emission lines of $\mathrm{H} \alpha, \mathrm{H} \beta,[\mathrm{N}$ II] $\lambda 6583$, and [O II] $\lambda 3727$. From the N2 diagnostic of Pettini \& Pagel (2004), we estimate an oxygen abundance of $\log (\mathrm{O} / \mathrm{H})+12=8.8 \pm 0.1$. Using the N202 diagnostic of Kewley \& Dopita (2002), we measure $\log (\mathrm{O} / \mathrm{H})+12=9.0 \pm 0.1$. These two values are consistent given the well-known offset between the diagnostics (Kewley \& Ellison 2008).

Adopting a solar metallicity of $\log (\mathrm{O} / \mathrm{H})_{\odot}+12=8.69$ (Asplund et al. 2005) on the PP04 scale, our measurement suggests that the environment of SN 2011ei has an approximately solar metallicity. This puts SN 2011ei on the high end of the observed distribution of SN IIb explosion site metallicities (see, e.g., Kelly \& Kirshner 2012; Modjaz et al. 2011; Sanders et al. 2012).

\section{RADIO AND X-RAY DIAGNOSTICS}

\subsection{Radio Light Curves, Forward Shock, and Magnetic Field}

While optical data probe the thermal emission from the slow-moving bulk ejecta, non-thermal synchrotron emission in the radio is produced as the forward shock races ahead and dynamically interacts with the CSM. Radio observations can thus provide unique information about the progenitor star's mass-loss history several years to centuries immediately prior to explosion.

We show the observed radio light curves of SN 2011ei in Figure 16. The radio light curves show flux density variations of a factor of $\sim 3$ with a $5 \mathrm{GHz}$ peak spectral luminosity, $L_{v} \approx 5.48 \times 10^{26} \mathrm{erg} \mathrm{s} \mathrm{Hz}^{-1}$, approximately 23 days after the explosion. The peak radio emission is fairly weak compared to the majority of SNe IIb and Ibc, but comparable 


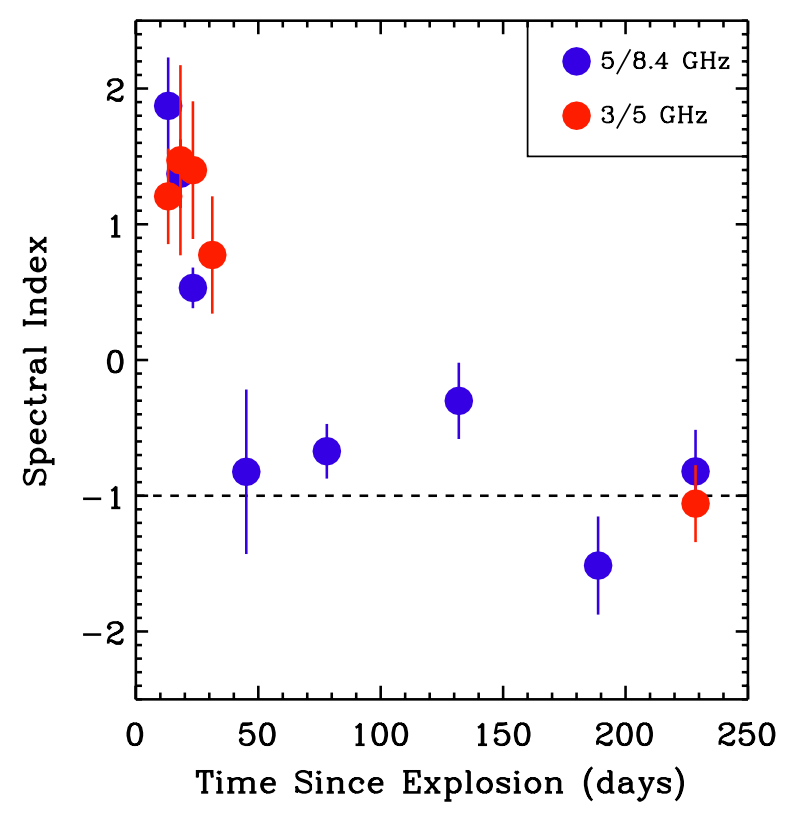

Figure 17. Evolution of the radio spectral indices spanning 3-8.4 GHz are shown. The early radio emission is optically thick, $F_{v} \propto v^{2}$, but gradually evolves to an optically thin spectrum, $F_{v} \propto v^{-1}$.

(A color version of this figure is available in the online journal.)

to, e.g., SN 2011dh, SN 2008ax, and SN 1996cb (Soderberg et al. 2012; Krauss et al. 2012; Chevalier \& Soderberg 2010). Approximately 40 days after the explosion, the radio emission decays steeply at all frequencies, then rises again to a second maximum $\sim 2$ months later.

As the forward shock expands into the surrounding environment, electrons are accelerated to relativistic velocities with a distribution, $N\left(\gamma_{e}\right) \propto \gamma_{e}^{-p}$, where $\gamma_{e}$ is the Lorentz factor of the particles. Amplified magnetic fields cause the shocked electrons to gyrate and emit synchrotron emission detectable in the $\mathrm{cm}$ band on a timescale of days after the explosion (Chevalier 1982). A low-frequency spectral turnover has been observed for radio $\mathrm{SNe}$ Ibc and IIb attributed to synchrotron self-absorption defining a spectral peak, $v_{a}$, and the spectrum is characterized by $F_{v} \propto v^{5 / 2}$ below $v_{a}$ and $F_{v} \propto v^{-(p-1) / 2}$ above $v_{a}$. At the epoch $v_{a}$ crosses each observing band, the radio light curve for that band reaches maximum intensity. The frequency and brightness at which the peak in the radio spectrum occurs allows us to calculate the radius of the emitting region, which is bound by the forward shock, and thus the average expansion velocity of the SN (Chevalier \& Fransson 2006).

In Figure 17, we show the radio spectral indices in adjacent bands spanning 3-8 GHz for SN 2011ei. The early radio emission is optically thick with $F_{v} \propto v^{2}$ while the later emission is optically thin with approximately $F_{v} \approx v^{-1}$, implying $p \approx 3$. As shown in Figure 16, the $5 \mathrm{GHz}$ light curve peaks on 2011 August 17 (i.e., time to peak since explosion, $t_{p} \approx 23$ days) with a flux density of $F_{v, p} \approx 560 \mu \mathrm{Jy}$. Adopting $p=3$, we estimate the time-averaged velocity, $\bar{v} \equiv R / \Delta t$, of the forward shock to be

$$
\begin{aligned}
\bar{v} \approx 0.14 c & \times\left(\epsilon_{e} / \epsilon_{B}\right)^{-1 / 19}\left(F_{\nu, p} / \mathrm{mJy}\right)^{9 / 19} \\
& \times(d / 10 \mathrm{Mpc})^{18 / 19}\left(v_{p} / 5 \mathrm{GHz}\right)^{-1} \\
& \times\left(t_{p} / 10 \text { days }\right)^{-1},
\end{aligned}
$$

or $\bar{v} \approx 0.13 c$ (see Chevalier \& Fransson 2006).
Here we have assumed that half of the volume enclosed by the forward shock is producing synchrotron emission, ${ }^{33}$ and $\epsilon_{e}$ and $\epsilon_{B}$ represent the efficiency of the shock wave in accelerating electrons and amplifying magnetic fields, respectively. We have further assumed that the particles and magnetic field are in equipartition (defined as $\epsilon_{e}=\epsilon_{B}$ ). The inferred shock wave velocity for SN 2011ei is in line with the velocities derived for $\mathrm{SNe}$ Ibc and compact progenitor SNe IIb (Chevalier \& Soderberg 2010; Soderberg et al. 2012), and higher than those inferred for extended progenitor SNe IIb and SNe of Type IIP (Chevalier 1998).

Following Chevalier \& Fransson (2006), we find the strength of the amplified magnetic field $(B)$ for the radio emitting material to be

$$
\begin{aligned}
B \approx 0.68 & \times\left(\epsilon_{e} / \epsilon_{B}\right)^{-4 / 19}\left(F_{v, p} / \mathrm{mJy}\right)^{-2 / 19} \\
& \times(d / 10 \mathrm{Mpc})^{-4 / 19}\left(v_{p} / 5 \mathrm{GHz}\right) \mathrm{G},
\end{aligned}
$$

or $B \approx 0.6 \mathrm{G}$ for equipartition.

\subsection{Mass-loss Rate and Evidence for an Inhomogeneous Wind}

Finally, we consider the mass-loss rate of the progenitor system. As shown in Figure 16, the radio light curves for SN 2011ei do not decline as a power law following maximum light, but instead show evidence for a secondary maximum at $\approx 80$ days post-outburst. As noted in Chevalier \& Soderberg (2010), secondary maxima are common among $\mathrm{SNe}$ IIb. Assuming the dominant absorption process is synchrotron self-absorption as suggested by Figure 17, the time averaged mass-loss rate $\dot{M}$ from the progenitor star can be estimated as

$$
\begin{aligned}
\dot{M} \approx & 0.38 \times 10^{-5} M_{\odot} \mathrm{yr}^{-1} \\
& \times\left(\epsilon_{B} / 0.1\right)^{-1}\left(e_{e} / e_{B}\right)^{-8 / 19}\left(F_{v, p} / \mathrm{mJy}\right)^{-4 / 19} \\
& \times(d / 10 \mathrm{Mpc})^{-8 / 19}\left(v_{\mathrm{p}} / 5 \mathrm{GHz}\right)^{2}\left(\mathrm{t}_{\mathrm{p}} / 10 \text { days }\right)^{2}
\end{aligned}
$$

for a wind velocity of $v_{w}=1000 \mathrm{~km} \mathrm{~s}^{-1}$ typical for a W-R star (Chevalier \& Fransson 2006).

For reasonable values of $\epsilon_{e}=e_{B}=0.1$ we find $\dot{M} \approx$ $1.4 \times 10^{-5} M_{\odot} \mathrm{yr}^{-1}$. We note that this is a lower bound on the inferred mass-loss rate from the progenitor system. Lower values of the partition fractions and deviations from equipartition (see, e.g., SN 1993J; Fransson \& Björnsson 1998) only serve to increase $\dot{M}$. The mass-loss rate for SN 2011ei is similar to within an order of magnitude to that inferred for other $\mathrm{SNe} \mathrm{IIb}$ and Ibc.

As seen in Figure 18, radio light curve variations observed similar to those observed in SN 2011ei have been detected for other SNe IIb including SN 2001ig (Ryder et al. 2004) and SN 2008ax (Roming et al. 2009), the broad-lined event SN 2003bg (Soderberg et al. 2006), as well as the gammaray burst (GRB) associated SN 1998bw (Kulkarni et al. 1998). These fluctuations are not dissimilar in both timescale and amplitude, and have been reasonably explained in terms of density modulations in the pre-explosion environment shaped by the progenitor system (Ryder et al. 2004; Soderberg et al. 2006; Roming et al. 2009).

\footnotetext{
33 Very long baseline interferometry radio images of SN 1993J suggested a spherical shell structure with a shell thickness $20 \%-33 \%$ of the outer radius (Bietenholz et al. 2010a; Martí-Vidal et al. 2011), in which case 49\%-70\% of the volume enclosed by the forward shock is filled with radio emission. However, possible small-scale clumpiness within this geometry could further lower the effective radio-emitting volume.
} 


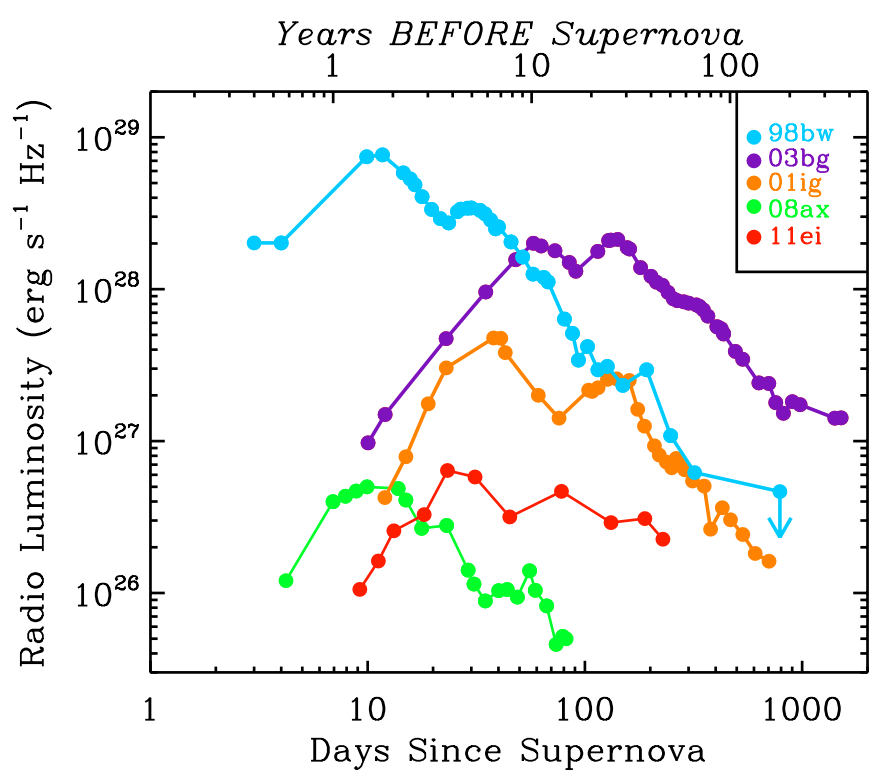

Figure 18. $5 \mathrm{GHz}$ light curve for SN 2011ei is compared to those of other radio supernovae including the Type IIb SNe 2001ig (Ryder et al. 2004), SN 2003bg (Soderberg et al. 2006), SN 2008ax (Roming et al. 2009), and the GRB-associated Type Ic SN 1998bw (Kulkarni et al. 1998). Bottom $x$-axis represents days since supernova outburst, and the top $x$-axis represents inferred times of CSM density modulations in years previous to outburst (assuming $v_{w}=1000 \mathrm{~km} \mathrm{~s}^{-1}$ ). Each of these $\mathrm{SNe}$ shows variable radio emission that deviates from the expected decay as roughly $F_{v} \propto t^{-1}$. In particular, all of these events show second maxima within a few months of the explosion.

(A color version of this figure is available in the online journal.)

We adopt a model in which the modulations in the radio light curve are similarly due to circumstellar density variations. As shown in Wellons et al. (2012), the optically thin synchrotron emission scales with the number density of shocked electrons as $F_{v} \propto n_{e}^{2}$ for $p=3$. Thus, assuming a constant compression factor by the forward shock, we find that the circumstellar density modulations scale similarly. Given the factor of $\sim 3$ in flux density modulations observed for SN 2011ei, we infer a factor of $\sim 2$ jump in the circumstellar density at a radius $r \sim 2 \times 10^{16} \mathrm{~cm}$. Attributing this effect to a variation in the progenitor wind of the progenitor star implies an ejection timescale of $\sim 7\left(v_{w} / 10^{3} \mathrm{~km} \mathrm{~s}^{-1}\right)$ yr prior to outburst.

It is intriguing to note that radio modulations have also been observed in relativistic, engine-driven $\mathrm{SNe}$ including GRB-SN 1998bw (Kulkarni et al. 1998; Li \& Chevalier 1999) and SN 2009bb (Bietenholz et al. 2010b). In these cases, the flux density modulations have been attributed to energy injection from the central engine. However, given the resemblance to the observed compact progenitor Type IIb radio modulations, we speculate that CSM density fluctuations on radial scales of $\lesssim 10^{17} \mathrm{~cm}$ may be common among stripped-envelope SN explosions.

\subsection{Limits on Inverse Compton X-Ray Emission}

For hydrogen-stripped SNe exploding in low-density environments, the dominant X-ray emission mechanism during the first weeks to a month after the explosion is inverse Compton (IC; Björnsson \& Fransson 2004; Chevalier \& Fransson 2006). In this framework, X-ray photons originate from the up-scattering of optical photons from the SN photosphere by a population of electrons accelerated to relativistic speeds by the SN shock. X-ray IC depends on the density structure of the SN ejecta, the structure of the CSM, and the details of the electron dis- tribution responsible for the up-scattering, but does not require any assumption on magnetic field related parameters and it is not affected by possible uncertainties on the SN distance. Thus, limits on X-ray emission can provide information on the preSN mass-loss history of the progenitor independent of our radio analysis.

Using the reconstructed bolometric luminosity and the derived explosion parameters $\left(M_{e j} \sim 1.6 M_{\odot}\right.$ and $E_{k} \sim 2.5 \times$ $10^{51} \mathrm{erg}$ ), and adopting the formalism discussed in Margutti et al. (2012), we estimated the progenitor mass-loss rate via X-ray IC. We assumed (1) the fraction of energy going into relativistic electrons was $\epsilon_{e}=0.1$ as indicated by well-studied SN shocks (Chevalier \& Fransson 2006), (2) a power-law electron distribution $N\left(\gamma_{e}\right)=n_{0} \gamma_{e}^{-p}$ with $p=3$ as indicated by radio observations of SNe Ibc (Chevalier \& Fransson 2006), (3) a wind-like CSM that follows $\rho_{\mathrm{CSM}} \propto R^{-2}$ as expected from a star which has been losing material at at average rate $\dot{M}$, and (4) that the outer density structure of the ejecta scales as $\rho_{\text {SN }} \propto R^{-n}$ with $n \sim 10$ (see, e.g., Matzner \& McKee 1999; Chevalier \& Fransson 2006). Given these assumptions, the Chandra upper limit on X-ray emission of $7.6 \times 10^{-4}$ counts s $^{-1}$ in the $0.5-8 \mathrm{keV}$ band implies $\dot{M}<2 \times 10^{-4} M_{\odot} \mathrm{yr}^{-1}$, for wind velocity $v_{w}=1000 \mathrm{~km} \mathrm{~s}^{-1}$. This calculation is consistent with the properties derived from our radio analysis (Section 5.2).

\section{DISCUSSION}

\subsection{The Progenitor of SN 2011ei}

The early onset of helium-rich features in the optical spectra of SN 2011ei and the evolution of its bolometric light curve are consistent with model explosions of He-core stars with pre-SN masses of 3-4 $M_{\odot}$ (Shigeyama et al. 1990; Woosley et al. 1995). The initial presence and subsequent rapid disappearance of hydrogen suggests the progenitor star still retained a thin hydrogen envelope of mass $<0.1 \quad M_{\odot}$ immediately prior to explosion.

Our inferred Ni mass of $\approx 0.04 M_{\odot}$ for SN 2011ei is on the low end of $\mathrm{Ni}$ masses derived for other stripped-envelope events $\left(\sim 0.05-0.15 M_{\odot}\right.$; Taubenberger et al. 2011). A low Ni mass yield is consistent with the explosions of lower-mass $\mathrm{He}$ star progenitors that have small iron cores $\left(\lesssim 0.15 M_{\odot}\right)$ and eject considerably less material than do their larger counterparts (Shigeyama et al. 1990; Hachisu et al. 1991). Lower-mass He stars are also predicted to undergo more extensive mixing than higher-mass stars do (Hachisu et al. 1991). The helium and iron expansion velocities determined from our synthetic spectral fits to SN 2011ei are quite similar (see Figure 9), and thus are in agreement with the emitting ejecta being well mixed.

Several properties of W-R stars make them plausible candidate progenitors for SN 2011ei: (1) W-R stars are believed to be likely progenitors of at least some $\mathrm{SNe}$ IIb and Ibc (Heger et al. 2003), (2) some subtypes of W-R stars (e.g., the WN class) have an observable amount of hydrogen at their surfaces (Hamann et al. 1991), (3) the radio-derived mass-loss rate of SN 2011ei is consistent with the observed rates for Galactic W-R stars $\left(10^{-4} \lesssim \dot{M} \lesssim 10^{-6} M_{\odot} \mathrm{yr}^{-1}\right.$; Hamann et al. 2006), and (4) the progenitor radius inferred from the early photometry $\left(R_{*} \lesssim 1 \times 10^{11} \mathrm{~cm}\right)$ is consistent with those of W-R stars (Crowther 2007). The putative W-R star could have evolved originally from either a single star with a high main-sequence mass of $\sim 25 M_{\odot}$, or a lower-mass star with main-sequence mass $\sim 10-15 M_{\odot}$ in an interacting binary system. 
The physical origin of the radio light curve variations and the inferred inhomogeneous wind is not clear. Interestingly, not all radio $\mathrm{SNe}$ show this behavior. For instance, several well-studied examples exhibit smooth light curve evolutions, including the Type IIb SNe 1993J and 2011dh (van Dyk et al. 1994; Bartel et al. 2002; Krauss et al. 2012), and the Type Ic SN 2003L (Soderberg et al. 2005). Thus, some aspect of the progenitors of stripped-envelope events like SN 2011ei (see Figure 18) produces variability in the winds of the progenitor systems on the timescale of $\sim 10 \mathrm{yr}$.

Luminous blue variables (LBVs) have been suggested as possible progenitor stars given that they share many characteristics of W-R stars and can undergo occasional giant eruptive events that play a major role in removing the $\mathrm{H}$-rich envelope on timescales that are consistent with SN 2011ei's radio light curve variability (see, e.g., Kotak \& Vink 2006). However, SNe IIb and Ib progenitors appear to be less massive and more compact than LBVs (Soderberg et al. 2006). Ultimately, although LBVs may not represent the evolutionary state immediately prior to explosion, LBV-like eruptions may provide the dominant method of stripping the H-rich envelope at low metallicity for W-R stars (Crowther 2007).

\subsection{Classification Confusion: IIb versus Ib}

At the earliest epochs observed, SN 2011ei exhibited emission properties consistent with a Type II classification. However, by the time of maximum light, SN 2011ei closely resembled many SNe Ib (see Figure 10). If the first optical spectra of SN 2011ei had only been obtained near maximum light, i.e., about one week after we in fact obtained the first spectrum, its early $\mathrm{H} \alpha$ emission would have been missed and the SN likely classified as a Type Ib event.

Two important lessons come from this rapid spectral evolution. One is that absorption around $6250 \AA$ in $\mathrm{SNe} \mathrm{Ib}$ can be interpreted as a clear signature of hydrogen. High-velocity hydrogen has long been suspected in $\mathrm{SNe}$ Ib spectra, but direct identification has been largely circumstantial because it has most often only been observed as absorption in the region of $\mathrm{H} \alpha$. However, there are now at least two other well-observed cases where the transition of an $\mathrm{H} \alpha$ profile showing broad emission has been monitored through to conspicuous absorption around 6250 Å: SN 2007Y (Stritzinger et al. 2009) and SN 2008ax (Chornock et al. 2011).

The second lesson is that the rapid disappearance of $\mathrm{H} \alpha$ emission in the early spectra of SN 2011ei underscores a classification ambiguity. Namely, in some cases, whether an event is classified as Type Ib or IIb depends on how early the first spectrum is obtained. Consequently, criteria that take into account the possibility of temporal selection effects should be developed to distinguish between $\mathrm{SNe} \mathrm{IIb}$ and $\mathrm{Ib}$. An unambiguous classification scheme is crucial if precise rates of $\mathrm{SNe} \mathrm{IIb}$ versus $\mathrm{Ib}$ events are to be estimated.

One possible spectral diagnostic for distinguishing between $\mathrm{SNe} \mathrm{IIb}$ and $\mathrm{Ib}$ may be the relative strength of the two absorption features around 6250 and $5700 \AA$. In Figure 19, spectra of SN 2011ei and the Type Ib SN 1998dt (Matheson et al. 2001) obtained around the time of maximum light are plotted. Both share many similar spectral features outside of the conspicuous $\mathrm{H} \alpha$ absorption only observed in SN 2011ei. Also plotted in Figure 19 are later-epoch spectra of SN 2011ei and the prototypical Type Ib SN 1999dn (Matheson et al. 2001). Again, all spectral features are largely identical outside of absorptions around $\mathrm{H} \alpha$ and $\mathrm{H} \beta$ only seen in SN 2011ei. This

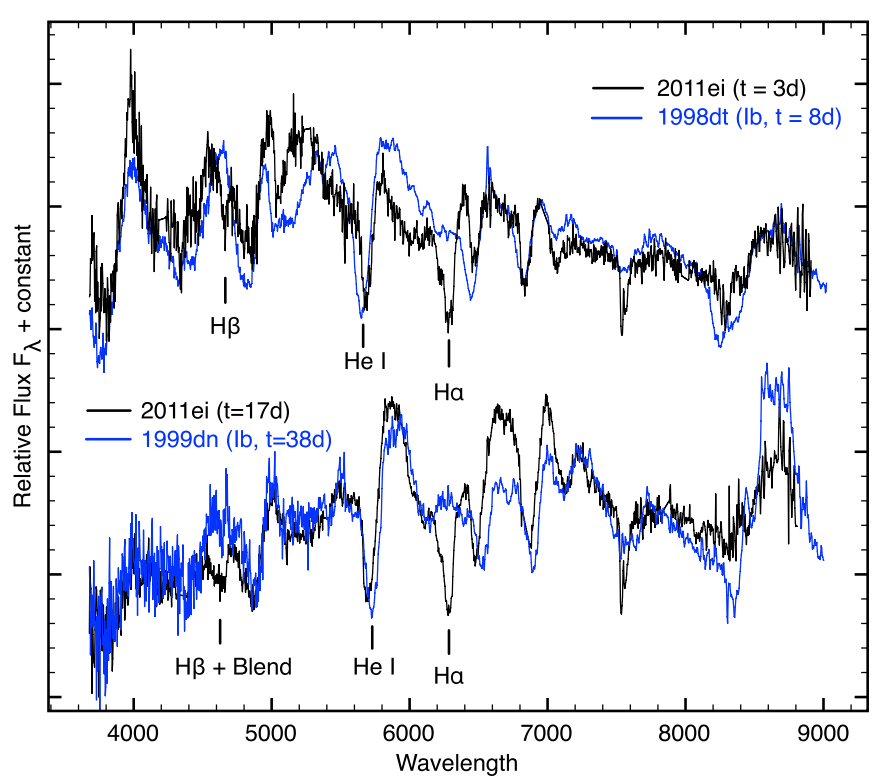

Figure 19. Spectra of SN 2011ei and the Type Ib SNe 1998dt and 1999dn (Matheson et al. 2001). The relative strength of He I $\lambda 5876$ absorption (observed near $5700 \AA$ ) to that of $\mathrm{H} \alpha$ absorption (observed near $6250 \AA$ ) could be used as a diagnostic to distinguish between Type IIb and $\mathrm{Ib}$ events.

(A color version of this figure is available in the online journal.)

pattern is observed in other SNe IIb as well; e.g., SN 1993J and SN 2001ig (see Figure 14). Thus, a Type IIb event may be distinguished from a Type Ib by an $\mathrm{H} \alpha$ absorption comparable to $\mathrm{He}$ I $\lambda 5876$ around the time of maximum light. This could be defined explicitly as $\mathrm{EW}(\mathrm{H} \alpha) / \mathrm{EW}\left(\mathrm{He}_{\mathrm{I}} \lambda 5876\right) \gtrsim 0.5$ within a few weeks of maximum.

\section{CONCLUSIONS}

We have presented X-ray, UV/optical, and radio observations of the He-rich, stripped-enveloped, core-collapse SN 2011ei beginning within $\sim 1$ day of explosion. The key findings of our analyses can be summarized as follows.

1. SN 2011ei was a relatively faint supernova with properties consistent with the outburst of an He-core star that suffered modest episodic mass loss. SN 2011ei was one of the least luminous stripped-envelope SNe observed to date. Our estimate of its ${ }^{56} \mathrm{Ni}$ mass (between 0.03 and $0.05 M_{\odot}$ ) places it on the extreme low end of ${ }^{56} \mathrm{Ni}$ masses estimated for other SNe Type IIb and Ibc. Radio light curve fluctuations in SN 2011ei suggest punctuated mass-loss episodes prior to outburst. These mass-loss variations are similar to those seen in a subset of other radio SNe and complicate the standard picture of smooth radiation-driven stellar winds. We suggest that a 3-4 $M_{\odot}$ W-R star of radius $R_{*} \lesssim$ $1 \times 10^{11} \mathrm{~cm}$ that retained a thin hydrogen envelope immediately prior to outburst is a plausible candidate progenitor for SN 2011ei.

2. High-velocity hydrogen is not rare in SNe Ib. Identification of hydrogen in Type Ib spectra has been largely circumstantial because it has most often only been observed as absorption around $\mathrm{H} \alpha$. In SN 2011ei, however, the transition of hydrogen emission through to absorption was closely monitored, which allowed an unambiguous identification of its origin. The existence of this transition lends support to the notion of a spectroscopic sequence bridging SNe IIb, $\mathrm{SNe} \mathrm{Ib}$ that have deep $\mathrm{H} \alpha$ absorptions, and typical $\mathrm{SNe} \mathrm{Ib}$, 
via a common progenitor scenario potentially rooted in differences in the hydrogen envelope mass. Whether this has origins in binary interaction and/or strong stellar winds is not clear, but circumstantial evidence suggests stellar duplicity plays a role in at least some cases.

3. Time-dependent classifications of SNe IIb and Ib bias estimates of their explosion rates. Like SN 2007Y (Stritzinger et al. 2009) and SN 2008ax (Chornock et al. 2011), SN 2011ei was caught early enough to observe a rapid evolution from Type II to Type Ib features in its pre-maximum light spectra. While SNe IIb have traditionally been understood to undergo this transformation on the timescale of months, examples such as SN 2011ei establish that the metamorphosis can occur on the timescale of a few days. Consequently, how promptly an observation is made after the time of explosion is a significant factor in a Type $\mathrm{IIb}$ versus Ib classification, and this has implications in determining precise rates.

Study of SN 2011ei shows that defining SNe Ib solely on the absence of hydrogen can lead to a classification confusion. There is no clear separation of $\mathrm{SNe}$ IIb versus $\mathrm{Ib}$ for at least a subset of these events. Instead, it is a gradual transition depending on remaining hydrogen mass. Along similar lines, the current definition of SNe Ic as lacking both hydrogen and helium lines may require revision. It is common practice to model SNe Ic in terms of core-collapse in bare carbon-oxygen cores (Iwamoto et al. 1994; Foley et al. 2003; Mazzali et al. 2004). However, there have been many reports of hydrogen and helium in SNe Ic (Filippenko 1988, 1992; Filippenko et al. 1990; Jeffery et al. 1991; Branch et al. 2002, 2006), and many objects exhibit properties that bridge SNe Ib and Ic subtypes; e.g., SN 2008D (Soderberg et al. 2008) and SN 2009jf (Valenti et al. 2011).

Thus, similar temporal selection effects may be at the root of uncertain line identifications in $\mathrm{SNe} \mathrm{Ib}$ and Ic. We recommend that multi-wavelength data suites like the one presented here could help resolve these ambiguities. Investigations of new SNe Ibc should incorporate high-cadence ( $\sim 2$ days) spectroscopic and photometric monitoring in the UV/optical commencing within days of explosion, and be coordinated with follow-up $\mathrm{X}$-ray and radio observations. From these panchromatic data, unique information about the progenitor star's poorly understood evolutionary state and associated mass loss in the years immediately prior to SN outburst can be extracted. Accumulation of even just a handful of additional objects studied in this way could establish fresh new insights into the nature of stripped-envelope SN explosions.

We thank an anonymous referee for suggestions that improved the manuscript. R. Chornock provided helpful comments. Some of the observations reported in this paper were obtained with the Southern African Large Telescope (SALT), as well as the $6.5 \mathrm{~m}$ Magellan Telescopes located at Las Campanas Observatory, Chile. Additional observations were obtained at the Southern Astrophysical Research (SOAR) telescope, which is a joint project of the Ministério da Ciência, Tecnologia, e Inovação (MCTI) da República Federativa do Brasil, the U.S. National Optical Astronomy Observatory (NOAO), the University of North Carolina at Chapel Hill (UNC), and Michigan State University (MSU). Support for this work was provided by the National Aeronautics and Space Administration through Chandra Award Number 12500613 issued by the Chandra $\mathrm{X}$-ray Observatory Center, which is operated by the Smithsonian Astrophysical Observatory for and on behalf of the National
Aeronautics Space Administration under contract NAS8-03060. The National Radio Astronomy Observatory is a facility of the National Science Foundation operated under cooperative agreement by Associated Universities, Inc. L.C. is a Jansky Fellow of the National Radio Astronomy Observatory. G.P. acknowledges support from "proyecto regular UNAB" DI-28-11/R. All SAAO and SALT co-authors acknowledge the support from the National Research Foundation (NRF) of South Africa. A.B. was supported by a Marie Curie Outgoing International Fellowship (FP7) of the European Union (project number 275596). G.P., F.B., and J.A. acknowledge support provided by the Millennium Center for Supernova Science through grant P10-064-F (funded by "Programa Bicentenario de Ciencia y Tecnologia de CONICYT" and "Programa Iniciativa Cientifica Milenio de MIDEPLAN"). F.B. acknowledges support from FONDECYT through Postdoctoral grant 3120227. J.A. acknowledges support by CONICYT through FONDECYT grant 3110142, and by the Millennium Center for Supernova Science (P10-064-F), with input from the "Fondo de Innvacin para La Competitividad, de Ministerio de Economa, Fomento Y Turismo de Chile." Partially based on observations (program ID 184.D-1140) collected at the European Organisation for Astronomical Research in the Southern Hemisphere, Chile. S.B. is partially supported by the PRIN-INAF 2009 with the project "Supernovae Variety and Nucleosynthesis Yields." ESO VLT observations were taken within the GO program 089.D-0032 (PI: P. Mazzali) with UT1/FORS2. P.M. and E.P. acknowledge financial support from INAF PRIN 2011 and ASI-INAF grants I/088/06/0 and I/009/10/0. D.M, E.P., and P.M. thank the Institute of Nuclear Theory at University of Washington where part of this work was accomplished. This paper made extensive use of the SUSPECT database (http://www.nhn.ou.edu/ suspect/). IRAF is distributed by the National Optical Astronomy Observatory, which is operated by the Association of Universities for Research in Astronomy (AURA) under cooperative agreement with the National Science Foundation. PYRAF is a product of the Space Telescope Science Institute, which is operated by AURA for NASA. Dust maps were accessed via the NASA/IPAC Infrared Science archive at http://irsa.ipac.caltech.edu/applications/DUST/.

Facilities: SALT (RSS), Magellan:Baade (IMACS), Magellan:Clay (LDSS3), Swift (UVOT,XRT), CXO, SOAR (Goodman), NTT (EFOSC2), CTIO:PROMPT

\section{REFERENCES}

Aldering, G., Humphreys, R. M., \& Richmond, M. 1994, AJ, 107, 662

Anderson, J. P., Covarrubias, R. A., James, P. A., Hamuy, M., \& Habergham, S. M. 2010, MNRAS, 407, 2660

Anupama, G. C., Sahu, D. K., Deng, J., et al. 2005, ApJL, 631, L125

Arcavi, I., Gal-Yam, A., Kasliwal, M. M., et al. 2010, ApJ, 721, 777

Arcavi, I., Gal-Yam, A., Yaron, O., et al. 2011, ApJL, 742, L18

Asplund, M., Grevesse, N., \& Sauval, A. J. 2005, in ASP Conf. Ser. 336, Cosmic Abundances as Records of Stellar Evolution and Nucleosynthesis, ed. T. G. Barnes III \& F. N. Bash (San Francisco, CA: ASP), 25

Baron, E., Hauschildt, P. H., \& Branch, D. 1994, ApJ, 426, 334

Baron, E., Hauschildt, P. H., Branch, D., et al. 1995, ApJ, 441, 170

Bartel, N., Bietenholz, M. F., Rupen, M. P., et al. 2002, ApJ, 581, 404

Bartunov, O. S., Blinnikov, S. I., Pavlyuk, N. N., \& Tsvetkov, D. Y. 1994, A\&A, 281, L53

Benetti, S., Branch, D., Turatto, M., et al. 2002, MNRAS, 336, 91

Benetti, S., Turatto, M., Valenti, S., et al. 2011, MNRAS, 411, 2726

Bietenholz, M. F., Bartel, N., Rupen, M. P., et al. 2010a, in Proceedings of the 10th European VLBI Network Symposium and EVN Users Meeting (Manchester, UK), 57

Bietenholz, M. F., Brunthaler, A., Soderberg, A. M., et al. 2012, ApJ, 751, 125 Bietenholz, M. F., Soderberg, A. M., Bartel, N., et al. 2010b, ApJ, 725, 4

Bigelow, B. C., Dressler, A. M., Shectman, S. A., \& Epps, H. W. 1998, Proc. SPIE, 3355, 225 
Björnsson, C.-I., \& Fransson, C. 2004, ApJ, 605, 823

Branch, D. 1972, A\&A, 16, 247

Branch, D., Benetti, S., Kasen, D., et al. 2002, ApJ, 566, 1005

Branch, D., Jeffery, D. J., Young, T. R., \& Baron, E. 2006, PASP, 118, 791

Brown, P. J., Holland, S. T., Immler, S., et al. 2009, AJ, 137, 4517

Burgh, E. B., Nordsieck, K. H., Kobulnicky, H. A., et al. 2003, Proc. SPIE, 4841, 1463

Burrows, D. N., Hill, J. E., Nousek, J. A., et al. 2005, SSRv, 120, 165

Cappellaro, E., Mazzali, P. A., Benetti, S., et al. 1997, A\&A, 328, 203

Chevalier, R. A. 1982, ApJ, 259, 302

Chevalier, R. A. 1998, ApJ, 499, 810

Chevalier, R. A., \& Fransson, C. 2006, ApJ, 651, 381

Chevalier, R. A., \& Fransson, C. 2008, ApJL, 683, L135

Chevalier, R. A., \& Soderberg, A. M. 2010, ApJL, 711, L40

Chomiuk, L., \& Soderberg, A. 2011, ATel, 3532, 1

Chornock, R., Filippenko, A. V., Li, W., et al. 2011, ApJ, 739, 41

Chugai, N. N. 2000, AstL, 26, 797

Clemens, J. C., Crain, J. A., \& Anderson, R. 2004, Proc. SPIE, 5492, 331

Clocchiatti, A., Wheeler, J. C., Phillips, M. M., et al. 1997, ApJ, 483, 675

Crawford, S. M., Still, M., Schellart, P., et al. 2010, Proc. SPIE, 7737, 773725

Crockett, R. M., Eldridge, J. J., Smartt, S. J., et al. 2008, MNRAS, 391, L5

Crowther, P. A. 2007, ARA\&A, 45, 177

Deng, J. S., Qiu, Y. L., Hu, J. Y., Hatano, K., \& Branch, D. 2000, ApJ, 540,452

Dessart, L., Hillier, D. J., Livne, E., et al. 2011, MNRAS, 414, 2985

Drout, M. R., Soderberg, A. M., Gal-Yam, A., et al. 2011, ApJ, 741, 97

Eldridge, J. J., Izzard, R. G., \& Tout, C. A. 2008, MNRAS, 384, 1109

Ensman, L., \& Burrows, A. 1992, ApJ, 393, 742

Filippenko, A. V. 1988, AJ, 96, 1941

Filippenko, A. V. 1992, ApJL, 384, L37

Filippenko, A. V. 1997, ARA\&A, 35, 309

Filippenko, A. V., Matheson, T., \& Barth, A. J. 1994, AJ, 108, 2220

Filippenko, A. V., Matheson, T., \& Ho, L. C. 1993, ApJL, 415, L103

Filippenko, A. V., Porter, A. C., \& Sargent, W. L. W. 1990, AJ, 100, 1575

Folatelli, G., Contreras, C., Phillips, M. M., et al. 2006, ApJ, 641, 1039

Foley, R. J., Challis, P. J., Filippenko, A. V., et al. 2012, ApJ, 744, 38

Foley, R. J., Papenkova, M. S., Swift, B. J., et al. 2003, PASP, 115, 1220

Foley, R. J., Sanders, N. E., \& Kirshner, R. P. 2011, ApJ, 742, 89

Fransson, C., \& Björnsson, C.-I. 1998, ApJ, 509, 861

Fransson, C., \& Chevalier, R. A. 1989, ApJ, 343, 323

Fryer, C. L., Mazzali, P. A., Prochaska, J., et al. 2007, PASP, 119, 1211

Gehrels, N., Chincarini, G., Giommi, P., et al. 2004, ApJ, 611, 1005

Georgy, C. 2012, A\&A, 538, L8

Hachinger, S., Mazzali, P. A., Taubenberger, S., et al. 2012, MNRAS, 422, 70

Hachisu, I., Matsuda, T., Nomoto, K., \& Shigeyama, T. 1991, ApJL, 368, L27

Hamann, W.-R., Duennebeil, G., Koesterke, L., Wessolowski, U., \& Schmutz, W. 1991, A\&A, 249, 443

Hamann, W.-R., Gräfener, G., \& Liermann, A. 2006, A\&A, 457, 1015

Hamuy, M., Deng, J., Mazzali, P. A., et al. 2009, ApJ, 703, 1612

Hamuy, M., Maza, J., Pinto, P. A., et al. 2002, AJ, 124, 417

Hamuy, M., Suntzeff, N. B., Heathcote, S. R., et al. 1994, PASP, 106, 566

Hamuy, M., Walker, A. R., Suntzeff, N. B., et al. 1992, PASP, 104, 533

Harkness, R. P., Wheeler, J. C., Margon, B., et al. 1987, ApJ, 317, 355

Heger, A., Fryer, C. L., Woosley, S. E., Langer, N., \& Hartmann, D. H. 2003, ApJ, 591,288

Houck, J. C., \& Fransson, C. 1996, ApJ, 456, 811

Iwamoto, K., Nomoto, K., Hoflich, P., et al. 1994, ApJL, 437, L115

James, S., \& Baron, E. 2010, ApJ, 718, 957

Jeffery, D. J., Branch, D., Filippenko, A. V., \& Nomoto, K. 1991, ApJL, 377, L89

Jeffery, D. J., Ketchum, W., Branch, D., et al. 2007, ApJS, 171, 493

Kalberla, P. M. W., Burton, W. B., Hartmann, D., et al. 2005, A\&A, 440, 775

Kelly, P. L., \& Kirshner, R. P. 2012, ApJ, 759, 107

Kewley, L. J., \& Dopita, M. A. 2002, ApJS, 142, 35

Kewley, L. J., \& Ellison, S. L. 2008, ApJ, 681, 1183

Koribalski, B. S., Staveley-Smith, L., Kilborn, V. A., et al. 2004, AJ, 128, 16

Kotak, R., \& Vink, J. S. 2006, A\&A, 460, L5

Krauss, M. I., Soderberg, A. M., Chomiuk, L., et al. 2012, ApJL, 750, L40

Kulkarni, S. R., Frail, D. A., Wieringa, M. H., et al. 1998, Natur, 395, 663

Landolt, A. U. 1992, AJ, 104, 340

Landolt, A. U. 2007, AJ, 133, 2502

Lewis, J. R., Walton, N. A., Meikle, W. P. S., et al. 1994, MNRAS, 266, L27

Li, W., Leaman, J., Chornock, R., et al. 2011, MNRAS, 412, 1441

Li, Z.-Y., \& Chevalier, R. A. 1999, ApJ, 526, 716

Maeda, K., Kawabata, K., Mazzali, P. A., et al. 2008, Sci, 319, 1220

Margutti, R., Soderberg, A. M., Chomiuk, L., et al. 2012, ApJ, 751, 134
Martí-Vidal, I., Marcaide, J. M., Alberdi, A., et al. 2011, A\&A, 526, A142

Matheson, T., Filippenko, A. V., Barth, A. J., et al. 2000, AJ, 120, 1487

Matheson, T., Filippenko, A. V., Li, W., Leonard, D. C., \& Shields, J. C. 2001, AJ, 121,1648

Matzner, C. D., \& McKee, C. F. 1999, ApJ, 510, 379

Maund, J. R., Fraser, M., Ergon, M., et al. 2011, ApJL, 739, L37

Maund, J. R., Smartt, S. J., Kudritzki, R. P., Podsiadlowski, P., \& Gilmore, G. F. 2004, Natur, 427, 129

Maurer, I., Mazzali, P. A., Taubenberger, S., \& Hachinger, S. 2010, MNRAS, 409, 1441

Mazzali, P. A., Deng, J., Hamuy, M., \& Nomoto, K. 2009, ApJ, 703, 1624

Mazzali, P. A., Deng, J., Maeda, K., et al. 2004, ApJ, 614, 858

Mazzali, P. A., Kawabata, K. S., Maeda, K., et al. 2005, Sci, 308, 1284

Mazzali, P. A., Maurer, I., Stritzinger, M., et al. 2011, MNRAS, 416, 881

Mazzali, P. A., Nomoto, K., Patat, F., \& Maeda, K. 2001, ApJ, 559, 1047

Mazzali, P. A., Valenti, S., Della Valle, M., et al. 2008, Sci, 321, 1185

Milisavljevic, D., Fesen, R. A., Gerardy, C. L., Kirshner, R. P., \& Challis, P. 2010, ApJ, 709, 1343

Milisavljevic, D., Fesen, R., Nordsieck, K., et al. 2011, ATel, 3526, 1

Modjaz, M., Kewley, L., Bloom, J. S., et al. 2011, ApJL, 731, L4

Modjaz, M., Kirshner, R. P., Blondin, S., Challis, P., \& Matheson, T. 2008, ApJL, 687, L9

Modjaz, M., Li, W., Butler, N., et al. 2009, ApJ, 702, 226

Murphy, J. W., Jennings, Z. G., Williams, B., Dalcanton, J. J., \& Dolphin, A. E. 2011, ApJL, 742, L4

Nakar, E., \& Sari, R. 2010, ApJ, 725, 904

Nomoto, K. I., Iwamoto, K., \& Suzuki, T. 1995, PhR, 256, 173

Oke, J. B. 1990, AJ, 99, 1621

Parrent, J., Branch, D., Troxel, M. A., et al. 2007, PASP, 119, 135

Pastorello, A., Kasliwal, M. M., Crockett, R. M., et al. 2008, MNRAS, 389, 955

Perley, R. A., Chandler, C. J., Butler, B. J., \& Wrobel, J. M. 2011, ApJL, 739, L1

Pettini, M., \& Pagel, B. E. J. 2004, MNRAS, 348, L59

Podsiadlowski, P., Joss, P. C., \& Hsu, J. J. L. 1992, ApJ, 391, 246

Poole, T. S., Breeveld, A. A., Page, M. J., et al. 2008, MNRAS, 383, 627

Poznanski, D., Ganeshalingam, M., Silverman, J. M., \& Filippenko, A. V. 2011, MNRAS, 415, L81

Predehl, P., \& Schmitt, J. H. M. M. 1995, A\&A, 293, 889

Prieto, J. L., Stanek, K. Z., \& Beacom, J. F. 2008, ApJ, 673, 999

Puls, J., Vink, J. S., \& Najarro, F. 2008, A\&ARv, 16, 209

Qiu, Y., Li, W., Qiao, Q., \& Hu, J. 1999, AJ, 117, 736

Reichart, D., Nysewander, M., Moran, J., et al. 2005, NCimC, 28, 767

Richardson, D., Branch, D., \& Baron, E. 2006, AJ, 131, 2233

Richmond, M. W., Treffers, R. R., Filippenko, A. V., et al. 1994, AJ, 107, 1022

Roming, P. W. A., Kennedy, T. E., Mason, K. O., et al. 2005, SSRv, 120, 95

Roming, P. W. A., Pritchard, T. A., Brown, P. J., et al. 2009, ApJL, 704, L118

Ryder, S. D., Sadler, E. M., Subrahmanyan, R., et al. 2004, MNRAS, 349, 1093

Sanders, N. E., Soderberg, A. M., Levesque, E. M., et al. 2012, ApJ, 758, 132

Schlegel, D. J., Finkbeiner, D. P., \& Davis, M. 1998, ApJ, 500, 525

Shigeyama, T., Nomoto, K., Tsujimoto, T., \& Hashimoto, M.-A. 1990, ApJL, 361, L23

Shigeyama, T., Suzuki, T., Kumagai, S., et al. 1994, ApJ, 420, 341

Silverman, J. M., Mazzali, P., Chornock, R., et al. 2009, PASP, 121, 689

Smartt, S. J. 2009, ARA\&A, 47, 63

Smith, N., Li, W., Filippenko, A. V., \& Chornock, R. 2011, MNRAS, 412, 1522

Smith, N., \& Owocki, S. P. 2006, ApJL, 645, L45

Soderberg, A. M., Berger, E., Page, K. L., et al. 2008, Natur, 453, 469

Soderberg, A. M., Chevalier, R. A., Kulkarni, S. R., \& Frail, D. A. 2006, ApJ, 651,1005

Soderberg, A. M., Kulkarni, S. R., Berger, E., et al. 2005, ApJ, 621, 908

Soderberg, A. M., Margutti, R., Zauderer, B. A., et al. 2012, ApJ, 752, 78

Springob, C. M., Masters, K. L., Haynes, M. P., Giovanelli, R., \& Marinoni, C. 2009, ApJS, 182, 474

Spyromilio, J. 1994, MNRAS, 266, L61

Stritzinger, M., Hamuy, M., Suntzeff, N. B., et al. 2002, AJ, 124, 2100

Stritzinger, M., Mazzali, P., Phillips, M. M., et al. 2009, ApJ, 696, 713

Stritzinger, M., Morrell, N., Pignata, G., et al. 2011, CBET, 2777, 1

Sutherland, P. G., \& Wheeler, J. C. 1984, ApJ, 280, 282

Tanaka, M., Yamanaka, M., Maeda, K., et al. 2009, ApJ, 700, 1680

Taubenberger, S., Navasardyan, H., Maurer, J. I., et al. 2011, MNRAS, 413, 2140

Taubenberger, S., Valenti, S., Benetti, S., et al. 2009, MNRAS, 397, 677

Thomas, R. C., Nugent, P. E., \& Meza, J. C. 2011, PASP, 123, 237

Turatto, M., Benetti, S., \& Cappellaro, E. 2003, in Proc. ESO/MPA/MPE Workshop, From Twilight to Highlight: The Physics of Supernovae, ed. W. Hillebrandt \& B. Leibundgut (Berlin: Springer), 200 
Valenti, S., Benetti, S., Cappellaro, E., et al. 2008, MNRAS, 383, 1485

Valenti, S., Fraser, M., Benetti, S., et al. 2011, MNRAS, 416, 3138

Van Dyk, S. D., Li, W., Cenko, S. B., et al. 2011, ApJL, 741, L28

van Dyk, S. D., Weiler, K. W., Sramek, R. A., Rupen, M. P., \& Panagia, N. 1994, ApJL, 432, L115

Watson, D. 2011, A\&A, 533, A16

Wellons, S., Soderberg, A. M., \& Chevalier, R. A. 2012, ApJ, 752, 17

Willick, J. A., Courteau, S., Faber, S. M., et al. 1997, ApJS, 109, 333
Woosley, S. E., Eastman, R. G., Weaver, T. A., \& Pinto, P. A. 1994, ApJ, 429,300

Woosley, S. E., Langer, N., \& Weaver, T. A. 1995, ApJ, 448, 315

Yoon, S.-C., Woosley, S. E., \& Langer, N. 2010, ApJ, 725, 940

Zhang, Q., Hu, J. Y., Wang, L. F., Mazzali, P. A., \& Wang, Z. R. 1995a, MNRAS, 277,1115

Zhang, Q., Wang, L., Hu, J., Mazzali, P. A., \& Wang, Z. 1995b, AcApS, 15, 228

Zhang, Q., \& Wang, Z. R. 1996, A\&A, 307, 166 